\title{
ARTICLE
}

\section{Newton Trajectories for the tilted Frenkel-Kontorova Model}

\author{
W. Quapp, ${ }^{\mathrm{a}}$ and J. M. Bofill ${ }^{\mathrm{b}}$ \\ ${ }^{a}$ Mathematisches Institut, Universität Leipzig, PF 100920, D-04009 Leipzig, Germany \\ (ORCID: 0000-0002-0366-1408); \\ ${ }^{b}$ Departament de Química Inorgànica i Orgànica, Secció de Química Orgànica, \\ and Institut de Química Teòrica i Computacional, (IQTCUB), Universitat de Barcelona, \\ Martí i Franquès 1, 08028 Barcelona, Spain (ORCID: 0000-0002-0974-4618)
}

\section{ARTICLE HISTORY}

Compiled February 4, 2019

\begin{abstract}
Newton trajectories are used for the Frenkel-Kontorova model of a finite chain with free-end boundary conditions. We optimize stationary structures, as well as barrier breakdown points for a critical tilting force were depinning of the chain happens. These special points can be obtained straight forwardly by the tool of Newton trajectories. We explain the theory and add examples for a finite-length chain of a fixed number of $2,3,4,5$ and 23 particles.
\end{abstract}

\section{KEYWORDS}

Frenkel-Kontorova model, Tilting, Newton trajectory, Barrier breakdown point, Twist map

\section{Introduction}

A method in solid-state Physics is that one selects a one-dimensional subsystem in solitary confinement from a larger example of particles. The subsystem consists interacting elements, and one considers the remaining part as a substrate. The substrate acts by a fixed potential on the extracted subsystem. One example of such a model is the Frenkel-Kontorova (FK) model [1, 2] introduced in 1938 by Yakov Il'ich Frenkel and Tatyana Kontorova. The one-dimensional subsystem is represented by a discrete chain of atoms harmonically coupled with their nearest neighbors, while the impact of the fixed substrate on the one-dimensional subsystem can be described by a sinusoidal form. One supposes that the equilibrium distance associated with the inter-particle interaction may or may not coincide with the period of the substrate potential. It is a simple physical model with two competing scales. The model possesses an extraordinary simplicity. However, it has the ability to account for many nonlinear problems.

The concept has subsequently been involved in many applications of the model: tribology [3-5], charge-density waves conductors [6-9], charge transport in solids and on crystal surfaces [10], magnetic or ferro- and antiferromagnetic domain walls 
[11], magnetic superlattices [12], superconductivity [13-15], vortex matter [16-18], fractal spin glasses [19], Josephson junctions [20, 21], quantum algorithm [8], and H-bonded chains [1], just to name a few. Nowadays sliding friction forms a broad interdisciplinary research field that often involves the application of the FK model $[3,22,23]$. However, here we will understand static structural properties of condensed matter [24], but complex dynamical processes are moved to future research [25].

In many applications, one particularly interesting aspect of the FK model is its driven form. The FK model additionally exhibits a very rich behavior when it is subjected to an external tilting force. Here Newton trajectories (NTs) come into play. They describe for every tilted potential energy surface (PES) the curve of the displaced stationary points under the increasing external force. NTs are mathematical tools. They are curves on the given PES where at every curve point the gradient of the PES points into the same direction called the search direction [26]. They are static properties of the PES, and the understanding of aspects of the static PESs of the FK model is the aim of this work. Note that we can traditionally treat NTs for usual pathways over the PES describing a changing structure of the chain, like steepest descent, or other chemical reaction path curves.

The paper has the following Sections. In Sect. 2 we report on the FK model which is tilted in Sect.3. A short review of the theory of NTs is added in Sect. 4 and it is explained how NTs can be applied to the FK model. Sect. 5 enrolls a gallery of examples for different small chains with $2,3,4,5$, and 23 atoms. We get symmetric and asymmetric minimums and saddle points (SPs) of index 1 and 2, we get kinks, anti-kinks, and a Peierls barrier, and we explain in the case $N=23$ a breather. Sect. 5 is the main part of the paper. In Sect. 6 , we discuss the twist map often applied to the FK model (and our questions to its use). In Sect. 7 we discuss some symmetry relations. The end is a Conclusion. Data of the case $N=23$ are collected in a supplementary material (SM).

\section{The FK model}

\subsection{Model formula}

$\mathbf{x}=\left(x_{1}, \ldots, x_{N}\right)$ is a linear chain of $N$ discrete atoms. The positions $x_{i}$ are on an axis. For all atoms holds $x_{i}<x_{i+1}$. They are sorted in a fixed order. We treat a finite chain, thus $N$ is less than infinity [27-34]. The reason is that, from the point of view of our planet earth, no physical system is infinite. In contrast, an infinite chain is a pure mathematical construct. Additionally we believe that calculations of infinite chains were incorrectly done in parts, during the past forty years, see below, Sect.6.

A spring force acts with a force constant $k$ between the atoms which would results in a constant natural distance $a_{o}$ of the atoms. Without the side force, the end points of the chain are such that the average distance is $a_{o}=\left(x_{N}-x_{1}\right) /(N-1)$. Different 'natural' distances are a possible generalization [35-37], which we do not use here. A fixed on-site potential with a periodicity of $a_{s}$ acts on the atoms in concurrence with the springs. The on-site potential mimics a rigid non deformable substrate. The ratio $a_{o} / a_{s}$ is named the misfit parameter. The PES for the variable changes of the $x_{i}$ is 
the Frenkel-Kontorova model

$$
V(\mathbf{x})=v \sum_{i=1}^{N}\left[1-\cos \left(\frac{2 \pi x_{i}}{a_{s}}\right)\right]+\sum_{i=1}^{N-1} \frac{k}{2}\left[x_{i+1}-x_{i}-a_{o}\right]^{2} .
$$

We put the factor at the sinusoidal on-site potential, $v=1$, throughout the paper. Then the spring constant, $k$, is also the ratio of the strength of the on-site potential to that of the spring potential. Because $v>0$, the on-site potential will modulate the chain [38], and we will generally get another average spacing, $\tilde{a}_{o}$. All quantities referred to in this work are dimensionless.

\subsection{Derivations of the PES}

We obtain for the gradient of the PES of the FK model

$$
g_{i}(\mathbf{x})=\partial V(\mathbf{x}) / \partial x_{i}=2 \pi / a_{s} \sin \left(2 \pi x_{i} / a_{s}\right)+k\left[2 x_{i}-x_{i-1}-x_{i+1}\right]
$$

for $i=2, . ., N-1$ with the two special cases

$$
\begin{gathered}
g_{1}(\mathbf{x})=\partial V(\mathbf{x}) / \partial x_{1}=2 \pi / a_{s} \sin \left(2 \pi x_{1} / a_{s}\right)-k\left[x_{2}-x_{1}-a_{o}\right], \\
g_{N}(\mathbf{x})=\partial V(\mathbf{x}) / \partial x_{N}=2 \pi / a_{s} \sin \left(2 \pi x_{N} / a_{s}\right)+k\left[x_{N}-x_{N-1}-a_{o}\right] .
\end{gathered}
$$

Finally, the Hessian matrix is $[33,39]$ for $i=1, \ldots, N$

$$
H_{i, j}(\mathbf{x})=\partial g_{i}(\mathbf{x}) / \partial x_{j}=\partial^{2} V(\mathbf{x}) / \partial x_{i} \partial x_{j}
$$

which is tridiagonal and where the non-zero elements are

$$
\left.\begin{array}{c}
H_{i, i-1}(\mathbf{x})=H_{i, i+1}(\mathbf{x})=-k \\
H_{i, i}(\mathbf{x})=\left(2 \pi / a_{s}\right)^{2} \cos \left(2 \pi x_{i} / a_{s}\right)+\left(2-\delta_{i, 1}-\delta_{i, N}\right) k
\end{array}\right\}
$$

taking into account that $H_{1,0}(\mathbf{x})=H_{N, N+1}(\mathbf{x})=0$.

\subsection{Stationary forms of the FK chain}

There were different methods in the past to treat the search of minimums of the FK chain.

(1) The so-called gradient dynamics leads to a 2-dimensional map, the standard map [24]. Its result depends on the initial atom of the chain and usually does not lead to an equilibrium chain. There are discussions on the difference between global minimums, and other results of the map $[30,32,40]$ which are quite unclear. We illustrate the method (and our objections) by an example in Sect.6.

(2) Calculation of dynamical states by a wave equation, or a damped wave equation

[1]. The calculation does not give the exact minimum of the FK model. But ground 
states are localized at minimums. It is very interesting, but expensive.

(3) Iteration of an algebraic eigenvalue equation [41, 42]. Many iterations are needed (in examples $\approx 40$ ).

(4) A Monte Carlo simulation [29] leads to diverging relaxation time scales. It prevents the FK model from equilibrating all the way to its zero-temperature ground state. Another Monte Carlo simulation was applied in Ref.[37].

(5) We propose here the application of NTs as a new method for the FK model. For control reasons, we also use the optimization in Mathematica, as well as the iteration (1).

\section{Tilting of the FK model}

Additionally to the two forces of the FK model, we use an external, linear force in the ansatz $[1,2,30,36,43-52]$. We name the resulting PES an effective PES

$$
V_{F}(\mathbf{x})=V(\mathbf{x})-F \frac{1}{\sqrt{N}}(1, . ., 1)^{T} \cdot \mathbf{x} .
$$

The multiplication point between the $N$-dimensional direction vector $(1, . ., 1)^{T}$ and the $N$-variable $\mathbf{x}$ means the scalar product. $F$ is a factor, and $1 / \sqrt{N}$ is the normalization.

In electrical circumstances, the new term is named dc driving (dc: direct current) $[2,53]$. The force tilts the former on-site potential with the incline $F$. The standard direction $(1, . ., 1)^{T}$ is physically useful because it is the simplest kind to move the chain altogether. It is also mathematically well chosen because the ground line of the spring potential has this direction. (Compare the double-dashed line in Figs.1 and 6 below). However note that we have to change the search direction in the case $N=5$. If $F=0$, then a minimum structure for the chain will exist. But if $F$ is large enough there a minimum does not exist, at all [30]. It is easy to understand if we separate the combination of the on-site potential and the external force for every atom, $x_{i}$. It concerns

$$
1-\cos \left(\frac{2 \pi x_{i}}{a_{s}}\right)-F x_{i} / \sqrt{N} .
$$

It is a corrugated plane with the global slope $F / \sqrt{N}$ [1]. The derivation to $x_{i}$ gives the local slope which acts on this atom $i$

$$
\frac{2 \pi}{a_{s}} \sin \left(\frac{2 \pi x_{i}}{a_{s}}\right)-F / \sqrt{N} .
$$

If we estimate $F>2 \pi \sqrt{N} / a_{s}$ then the force is directed downwards. The coarse estimation holds for all atoms. It means that no global pinning can take place. Interesting is the so-called pinning-depinning transition, as well as the backward process [31]. The barrier for a depinning can be reduced in the case of a special misfit parameter between chain and substrate. It was coined superlubricity [54-59]. 
An alternating force vector is proposed in the direction $\frac{1}{\sqrt{N}}(1,-1, . ., \pm 1)^{T}$ if an electric field is used for the breaking of the symmetry of a state [60]. A chain of two different atoms $[37,39]$ or ions may also feel an alternating force. For computational reasons we can use any directional vector, $\mathbf{l}=\left(l_{1}, \ldots, l_{N}\right)^{T}$ with $|l|=1$, of the $N$-dimensional coordinate space for a search along a special NT, see the next Sect.4, and the application in the case $N=5$ below.

\section{Newton trajectories}

\subsection{The Definition}

The stationary points on the effective potential with general force vector $\mathbf{f}=$ $F\left(l_{1}, . ., l_{N}\right)^{T}$ satisfy the condition $\nabla_{\mathbf{x}} V_{F}(\mathbf{x})=\mathbf{0}$. Its minimums and SPs satisfy the vector equation

$$
\nabla_{\mathbf{x}} V_{F}(\mathbf{x})=\mathbf{g}(\mathbf{x})-\mathbf{f}=\mathbf{0}
$$

One searches a point where the gradient of the original PES, $\mathbf{g}(\mathbf{x})$, has to be equal to the force, $\mathbf{f}$. Such an ansatz is named Newton trajectory $[61,62]$ to the force, $\mathbf{f}$. The gradient of $V(\mathbf{x})$ is the inner force of the chain, $\mathbf{x}$, against changes of its structure. This force has to be equal to the external force, $\mathbf{f}$. Then the new chain is again in an equilibrium. We are at a stationary point of $V_{F}(\mathbf{x})$. The NT describes a curve of force-displaced stationary points (FDSPs) of the tilted PES under a different load, $F$ [26, 61-66]. Usually, the energy of minimums is increased, but the energy of SPs is lowered. This means that the barriers become lower.

Of course, an NT can be treated without the treatment of the physics of the external force in Eq.(7). One only needs an abstract search direction. Then any NT describes a connection between different stationary points of an index difference of one [67]. Following an NT is a method to search a next SP if a minimum is given, or vice versa.

Eq.(10) can be written in a projector form $[61,62]$

$$
\left(\mathbf{U}-1 \mathbf{l}^{T}\right) \mathrm{g}(\mathbf{x})=\mathbf{0}
$$

where $\mathbf{U}$ is the unit matrix and the $\mathbf{l}$-unit vector is the normalized direction of $\mathbf{f}$. The equation (11) means nothing else than that $\mathbf{g}$ and $\mathbf{l}$ are parallel. If we differentiate the projector Eq.(11) with respect to the parameter that characterizes the FDSPs curve, $s$ in $\mathbf{x}(s)$, we obtain with the Hessian, $\mathbf{H},[62,64]$

$$
\left(\mathbf{U}-1 \mathbf{l}^{T}\right) \mathbf{H}(\mathbf{x}) \frac{d \mathbf{x}}{d s}=\mathbf{0} .
$$

This is an expression of the tangent of the FDSPs curve. For the calculation, the continuous NT is approximated by $L$ node points. They should not be interchanged with the $N$ atoms of the chain. The $N$ atoms form a point in the $N$-dimensional configuration space. A curve of such points is the NT. And it is numerically treated by its $L$ nodes. Because we have the Hessian of the FK model at hand, see Eqs.(5) 
and (6), then Eq.(12) is a way to generate the NT of a successive tilting. We use for the calculations a predictor-corrector method. For the predictor we use the tangent of the NT with Eq.(12), or the growing string ansatz [68,69], the latter at least for the small chains in the examples. It is usable: if we are at any $\mathbf{x}_{\text {start }}$ point, then the next final point $\mathbf{x}_{e n d}$ of an NT in the $N$-dimensional configuration space can be at $x_{i}+a_{s}$ for all atoms $i=1, . ., N$. This is a point of the same energy like $\mathbf{x}_{\text {start }}$, and the next stationary points of the chain must be in between. Compare Fig. 6 below: not all stationary points are usually on one and the same branch of the NT. Thus, if the PES becomes more complicated then different NTs have to be tested. The growing string ansatz holds by the periodicity of the on-site potential of the PES (1). However, if the chain becomes variable then the standard direction $(1, \ldots, 1)^{T}$ often becomes obsolete for a good search direction. We choose the initial chain of nodes of the NT of interest by the straight line between the two initial points, $\mathbf{x}_{\text {start }}$ and $\mathbf{x}_{\text {end }}$. The tangent of Eq.(12) is employed for the corrector direction, back to the correct NT, with a Newton-Raphsen-like method [69].

Note that the following along an NT always uses a step in the $N$-dimensional coordinate space. Thus every search step moves all $N$ atoms of the chain. In contrast, the gradient steps of the twist map method (1) in Subsection 2.3 move one single atom from step to step behind the other, in the chain.

\subsection{Application of NTs to the FK model}

An equilibrium of the chain is such that the total force on each atom is zero [70]. Now we search for a minimum of the FK model. Here are the three steps:

(1) Build a natural chain with spacing $a_{o}$. Set, for example, $x_{1}=0$ for the initial atom, thus it is in the first well of the on-site potential, and set $x_{i}=(i-1) a_{o}$ for $i=2, . ., N$.

(2) Put the natural chain into the PES of Eq.(1), $V(\mathbf{x})$, and calculate the gradient $\mathbf{g}(\mathbf{x})$ with Eqs. (2) to (4).

(3) Form the normalized direction $\mathbf{l}$ along the negative gradient. It is the search direction of an NT which goes through the surface point $V(\mathbf{x})$. Follow the NT downhill to the next stationary state which usually is a minimum.

Such a minimum will always exist [71]. Each of the $N$ atoms of the chain will 'look' into (at least) one minimum well of the on-site potential. In the trivial case that $a_{o}=a_{s}$ one is finished. The natural chain is the global minimum. Usually, it is $a_{o} \neq a_{s}$. Then the minimization of the PES, Eq.(1), will move the atoms a little into their corresponding wells of the on-site potential down to an equilibrium with the spring forces, and the average spacing will become a value $\tilde{a}_{o}$ near the former $a_{o}$ (if $k>0$ what we always assume). The natural minimization excludes the assumption of periodic boundary conditions $[20,31]$

$$
x_{N}=x_{1}+2 a_{s} M
$$

with an appropriate integer $M$. The latter ansatz will fix $a_{o}$. However, in experiments with several tens of ions in a chain an inhomogeneous spacing was observed, in particular at the edges of the crystal [72]. But of course, the free ends of the chain are locked by the springs. 
If some of the atoms, say $x_{j}$ for some $j$, are at the beginning at a top of the on-site potential, then the other atoms left, or right from $x_{j}$ will pull them down to one of the both wells below the top. (See below an example with $N=23$ atoms.) In some special cases one can finish at a symmetric SP. Here one can give to one, or two, or all distances $a_{o}$ a tiny distortion. Then the NT finds the way downhill to the minimum.

The minimum is a point in the $N$-dimensional coordinate space. If the chain is here, it is fixed, for all times, if no other outer force moves it out of its state. No sliding or so can happen. The same applies if $N$ becomes larger and larger, compare Ref.[35]. Each of the $N$ atoms sits in its own minimum well of the on-site potential. And so each of the atoms must be moved out of the global minimum - for a hypothetical sliding. In some special configurations, compare the case $N=5$ below, there a fraction of atoms can sit at a top of the on-site potential [27, 73]. However, they are held at this 'strain-free' configuration by the springs to the other atoms inside the potential wells. Here too no sliding is possible of the full chain.

It is not always correct that the global minimum is unique [70]. The case $N=23$ below has an asymmetric global minimum. Of course there exits a mirror structure with the same energy. So we find here at least two equivalent global minimums.

\subsection{Barrier breakdown points - BBPs}

If one goes along the corresponding FDSPs curve with a given direction $\mathbf{l}$, then the magnitude of the force, $F$, starts with zero at a stationary point of $\mathrm{V}(\mathbf{x})$, and ends with zero at the final next stationary point. In between there has to be a maximum of $|\mathbf{g}|$. Here holds the condition $[65,74,75]$

$$
\operatorname{Det}(\mathbf{H}(\mathbf{x}))=0
$$

with the Hessian of the original PES, $V(\mathbf{x})$. This is the point where the gradient norm achieves a turning point. For every value of a tilting, $F$, on the path we get an effective PES, $V_{F}$. At the point with the property of Eq.(14) the effective $V_{F}(\mathbf{x})$ achieves a shoulder along the FDSPs path $[65,74,75]$. So to say, the tilting force is here at its 'limit'. The barrier of $V_{F}(\mathbf{x})$ decreases from the original PES barrier to zero. The point on the FDSPs curve is referred to as the barrier breakdown point (BBP). (We adapt here the similar use of such points which are named in Chemistry bond breaking point, BBP. In the FK model, no bonds break, but similar points on the PES exist.) The force which is to apply to meet the BBP on the corresponding effective PES is named the critical force, $F_{c}$, or the static frictional force. The effective PES has here a saddle-node bifurcation $[30,76]$. The force is so high that it causes the depinning of the chain, over the former barrier. Note that a barrier in an experiment will actually be surmounted before the BBP due to finite temperature effects $[77,78]$.

In order to find an optimal force for a BBP to be applied to the chain we need to know the direction of this force. Previous works by us demonstrated that this optimal direction can be rigorously and elegantly found using the NT-model [76, 86]. If we compare all NTs of a set which connect the same minimum and SP, then the NT which gives the lowest value of $F_{c}$ is the optimal $N T$, and the point of the intersection with the $\operatorname{Det}(\mathbf{H})=0$-manifold is named the optimal BBP $[65,75,86]$. The gradient of the original PES at a BBP gives the direction of the force to enforce the critical movement of the chain. The optimal BBP defines the lowest maximal force in magnitude and in a corresponding direction to be applied, in comparison to the FDSPs curves of other 
directions. The optimal BBP satisfies the vector equation $[65,75]$

$$
\mathbf{H}(\mathbf{x}) \mathbf{g}(\mathbf{x})=\mathbf{0} \quad \text { where } \quad \mathbf{g}(\mathbf{x}) \neq 0 .
$$

At the optimal BBP the gradient is an eigenvector of the Hessian matrix with eigenvalue zero. Such a point is on a gradient extremal (GE) [79-85]. At the optimal $\mathrm{BBP}$, the $\operatorname{Det}(\mathbf{H})=0$-manifold, the GE and the optimal regular NT meet. The optimal BBP is a stationary point on the function $|\mathbf{g}|$ through the actual equipotential curve crossed by the optimal NT. The GE and the NT are one-dimensional curves, but the condition $\operatorname{Det}(\mathbf{H})=0$ is a manifold in higher dimensions. An algorithm to locate optimal BBPs has been recently proposed [86]. We conclude the definition of BBPs with the remark that the barrier of the original PES, $V$, disappears on the effective PES, $V_{F_{c}}$, at the BBP point of the corresponding NT, if the critical amount of the force, $F_{c}$, is applied.

The expression of the optimal BBP, Eq.(15), has a special form for the FK-model because of the tridiagonal shape of the Hessian, Eqs.(5) and (6). Now Eq.(15) is mainly determined by the first and the last components of the g-vector, $g_{1}$ and $g_{N}$ and the diagonal elements of the $\mathbf{H}$ matrix. We get that the two border components of the gradient cannot be zero, at an optimal BBP point

$$
g_{1}\left(\mathbf{x}_{o p B B P}\right) \neq 0, \quad \text { and } \quad g_{N}\left(\mathbf{x}_{o p B B P}\right) \neq 0 .
$$

For a reasoning we take the general formula of the determinant of a tridiagonal symmetric matrix with rows of $(-k)$-entries in the secondary diagonals, compare a similar representation [33]. This has the recursive expressions with new symbols $\mathbf{H}(i)$

$$
\begin{aligned}
& \operatorname{Det}(\mathbf{H}(0))=1, \quad \operatorname{Det}(\mathbf{H}(1))=H_{1,1}, \\
& \operatorname{Det}(\mathbf{H}(2))=H_{2,2} H_{1,1}-k^{2}=H_{2,2} \operatorname{Det}(\mathbf{H}(1))-\operatorname{Det}(\mathbf{H}(0)) k^{2}, \\
& \ldots, \\
& \operatorname{Det}(\mathbf{H}(N))=H_{N, N} \operatorname{Det}(\mathbf{H}(N-1))-\operatorname{Det}(\mathbf{H}(N-2)) k^{2} .
\end{aligned}
$$

With the definitions at hand and with Eq.(15) we obtain an interesting and compact expression for the optimal BBP associated to the FK-model (1). We calculate the product between the $\mathbf{H}$ matrix of dimension $N \times N$ and the vector $\mathbf{g}$ of dimension $N$. The first row gives

$$
k g_{2}=H_{1,1} g_{1}=\operatorname{Det}(\mathbf{H}(1)) g_{1},
$$

the second row gives the following after multiplication with $k$

$$
k^{2} g_{3}=k H_{2,2} g_{2}-k^{2} g_{1}=H_{2,2} H_{1,1} g_{1}-k^{2} g_{1}=\operatorname{Det}(\mathbf{H}(2)) g_{1}
$$

and so on until

$$
k^{N-1} g_{N}=\operatorname{Det}(\mathbf{H}(N-1)) g_{1} .
$$

Finally substituting these expressions in the last row, we have 
$0=H_{N, N} g_{N}-k g_{N-1}$

and after multiplication with $k^{N-1}$ we get

$=H_{N, N} \operatorname{Det}(\mathbf{H}(N-1)) g_{1}-\operatorname{Det}(\mathbf{H}(N-2)) g_{1}=\operatorname{Det}(\mathbf{H}(N)) g_{1}$.

Thus the final expression is $\operatorname{Det}(\mathbf{H}(N)) g_{1}=0$. It is a very compact formula. Now $g_{1}=0$ implies that $g_{2}=g_{3}=\cdots=g_{N}=0$ which is the necessary condition of a stationary point, but it would be in contradiction to the condition of an optimal BBP that $\mathbf{g} \neq \mathbf{0}$. The formula is in accordance with the fact that the optimal BBP belongs to the manifold of points which satisfy the equality $\operatorname{Det}(\mathbf{H}(N))=0$ since $g_{1} \neq 0$. Thus the necessary and sufficient condition that a point $\mathbf{x}$ is an optimal BBP in the FK model is that the recursive relations Eqs.(17) to (19) are satisfied until $N$ and that $g_{1} \neq 0$.

A similar reasoning concerns the last component $g_{N} \neq 0$.

From the set of equations that characterizes an optimal BBP of the FK-model, $g_{1} \neq 0, g_{i}=\operatorname{det}(\mathbf{H}(i-1)) g_{1}$ for $i=2, \ldots, N$ and $\operatorname{det}(\mathbf{H}(N))=0$ one can derive the normalized optimal force, $\mathbf{f}_{n o f}$, at the BBP

$$
\mathbf{f}_{n o f}=\frac{1}{\sqrt{1+\sum_{i=1}^{N-1}\left(\operatorname{Det}(\mathbf{H}(i))^{2}\right.}}\left(\begin{array}{c}
1 \\
\operatorname{Det}(\mathbf{H}(1)) \\
\vdots \\
\operatorname{Det}(\mathbf{H}(N-1))
\end{array}\right) .
$$

The optimal force depends on the determinant factors of the $\mathbf{H}\left(\mathbf{x}_{o p B B P}\right)$ matrix. It is a result of the Catastrophe Theory on the FK-model (1). The normalized force vector, $\mathbf{f}\left(\mathbf{x}_{B B P}\right)$ of Eq. (20) is a more general vector since it is the eigenvector of the zero eigenvalue of the Hessian matrix of the FK-model. At any point of the manifold $\operatorname{Det}(\mathbf{H}(N))=0$, where $N$ is the dimension of the $\mathbf{x}$-space, the vector $\mathbf{f}\left(\mathbf{x}_{B B P}\right)$ is the eigenvector to the zero eigenvalue of this Hessian at the point $\mathbf{x}_{B B P}$ of the manifold.

\section{Simple Examples of FK Chains for Misfit $<1$}

\subsection{Example 1: 'Chain' of 2 Atoms [59, 87]}

To explain the formula (1), we study the case $N=2$ with $k=1, a_{o}=4 \pi / 3$ and $a_{s}=2 \pi$. The two atoms are named $x_{1}$ and $x_{2}$. Because $a_{o} \neq a_{s}$ there emerges a competition between the two nonlinear forces. The misfit parameter $a_{o} / a_{s}$ here is $2 / 3$. If we had $k=0$, or $a_{0}=a_{s}$, then the two atoms could be placed in two minimums of the on-site potential with the distance $2 \pi$. However, $k>0$ and $a_{0} \neq a_{s}$ disturb the location and produce a minimum anywhere in between because we assume $a_{0}<a_{s}$. Fig. 1 shows the PES with 20 equidistant contours with 0.5 units spacing (drawn by Mathematica, vers.11.2, like all other figures of this paper). We have included, for comparison, the ground line of the spring potential, by a double-dashed line. It always has the slope 1 (by definition of the spring potential), but the intersection with the $x_{2}$ axis is the parameter $a_{0}$. The values of the PES on this line are the original heights of the sinusoidal on-site potential. The pair $x_{1}-x_{2}$ here has the ideal distance $a_{0}$ of 


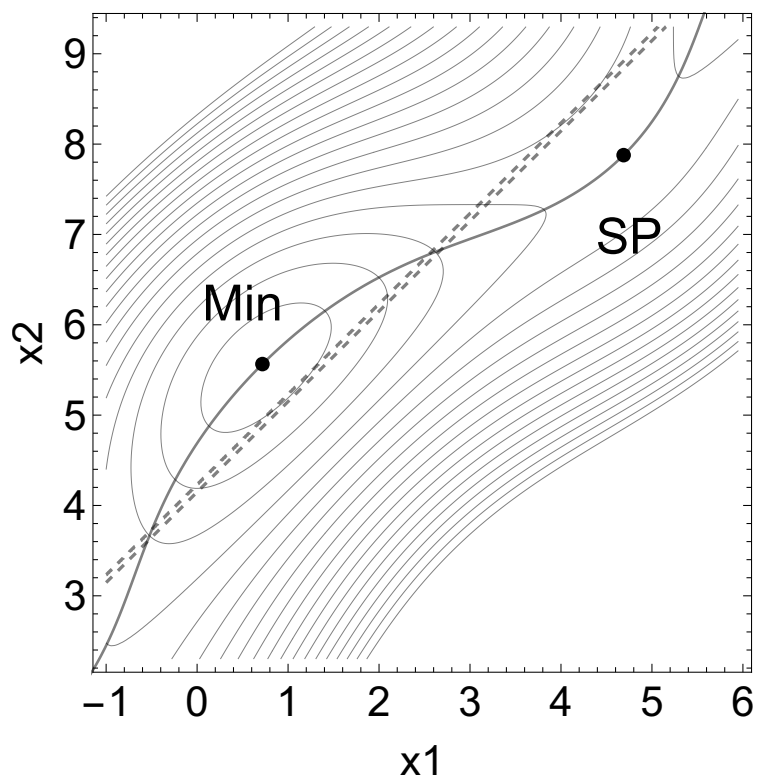

Figure 1. PES for a 'chain' with 2 atoms with locations $x_{1}$ and $x_{2}$ on a line. The minimum is at (0.72, 5.57) with $V=0.71$, and the SP is at $(4.69,7.88)$ with $V=2.55$. The energy difference is $\Delta V=1.83$ being a little lower than the assumed $\approx 2$ units [29]. The eigenvalues of the Hessian at the SP are -0.0237 and 1.98 . The curvature of the pathway over the SP is very slow. Any curve which connects Min and SP along the valley is a 'MEP'. The thick double-dashed line is the valley ground line of the trough of the spring potential.

the spring length. The spring potential increases orthogonally to this line. Minimums of the on-site potential which are far away are superimposed by the spring potential. So, in the cases of this example 1 , only one minimum energy path (MEP) remains meandering along the double-dashed line. The MEP in Fig. 1 is the NT to direction $(1,1)^{T}$.

The MEP is a path which is located in a valley of the PES. The path is a curve on the PES such that it is monotonically ascent from the initial minimum to the lowest SP and monotonically descent from this SP to the final minimum. Fore more than 40 years, the MEP is used in Chemistry [84, 88, 89]. Very often it is a synonym for the steepest descent from the SP of a reaction to a minimum. However, other curves which connect the minimum and the lowest SP can also serve for the MEP, like NTs without turning points [61].

Figure 2 schematically shows the minimum pair for the $N=2$ case. The movement of the two atoms out off the minimums of the on-site potential is caused by the spring between the two atoms. The location of the atoms is used on the profile of the cosine function to demonstrate the equilibrium of the FK model, in the case. The on-site potential pulls the atoms down into their minimum wells, but the spring force shortens the distance and so pulls the atoms uphill. The initial value $a_{o}=4.19$ is elongated by the on-site potential to 4.85 .

Figure 3 schematically shows the pair $x_{1}-x_{2}$ at the SP of the PES of Fig.1. It is a metastable state between two minimums. The location of the atoms is used on the profile of the cosine function to demonstrate the instable SP equilibrium of the FK model. It is an unstable 'anti-kink' $[29,53,90]$. Two atoms are compressed into one minimum of the on-site potential with a very short distance. The on-site potential 


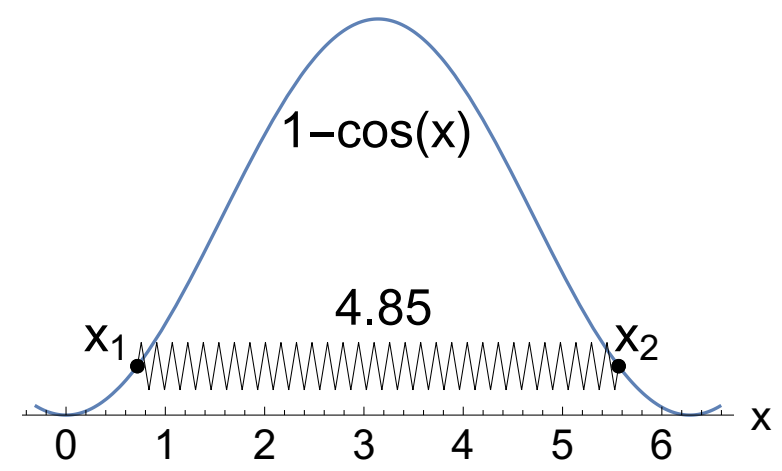

Figure 2. Schematic 'chain' with 2 atoms with minimum energy in relation to the on-site potential. The two atoms are artificially placed on the profile of the on-site potential, $1-\cos (x)$. Note that their real place is the projection to the axis.

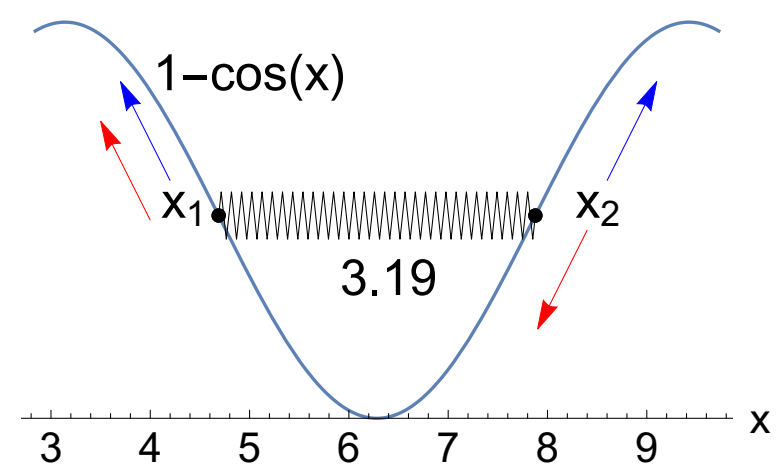

Figure 3. SP structure of the 'chain' with 2 atoms in relation to the on-site potential. Compare Figs. 1 and 2. The location of the pair on the on-site potential is labile. Any smallest push to one side moves it back to the minimum of Fig.2, or to the next periodic minimum in $2 \pi$ distance. The red arrows are the eigenvector to eigenvalue -0.024 along the SP valley, but the blue vectors are the eigenvector to eigenvalue 1.976 across the SP valley.

pulls the atoms down into one minimum, however, now the spring force extends the distance and so pulls the atoms uphill. The initial value $a_{o}=4.19$ is shortened by the on-site potential to 3.19. Note that the atoms of this SP structure are not placed on the top points of the on-site potential, as is generally assumed [91, 92]. The SP is a first example of an anti-kink. In the ground state, the atoms occupy the same wells as if would if uniformly distributed. An atom in a wrong well causes a local compression of the chain which costs energy [29]. The same holds for an additional gap, a kink, which stretches the chain. Historical details to kinks and anti-kinks can be found in Ref.[93]. The original German names for the kinks and breathers were 'translatorische und oszillatorische Eigenbewegungen'.

A successive tilting of the chain by an external force can cause a movement from the minimum to the SP and further to the next minimum. It would be the movement of the full chain along the axis. Thus, if one moves the pair $x_{1}-x_{2}$ along the on-site potential, it becomes clear from Fig.1 that this is only usefully possible along the one existing MEP which connects the consecutive minimums over the corresponding SPs. This pathway coarsely follows the straight minimum pathway of the spring potential. One always moves the connected pair. A tear into two single atoms is inhibited by the 
spring. Already forbidden is a 'location' of the two atoms in two minimums with a distance of $4 \pi$. However, the translation from one minimum to the next is not done by a uniform chain, (compare Ref.[27].) because the MEP is a bit curvilinear, here in Fig. 1.

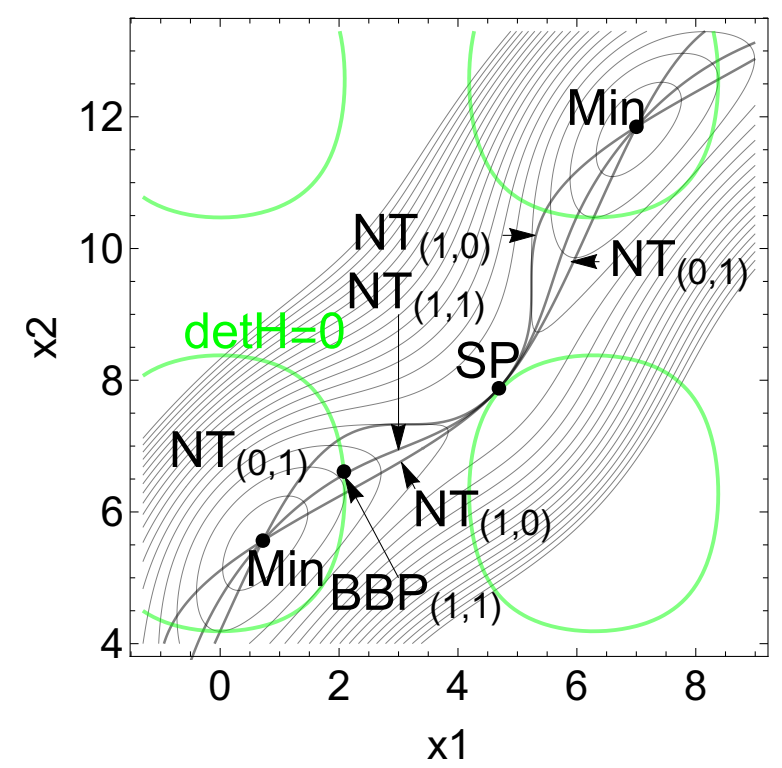

Figure 4. $\mathrm{BBP}$ on the $\mathrm{NT}_{(1,1)}$ for the 'chain' with 2 atoms in example 1. Its coordinates are (2.08,6.61). For comparison, also the two NTs to the axes directions are included, $\mathrm{NT}_{(1,0)}$ and $\mathrm{NT}_{(0,1)}$. On the green curves holds $\operatorname{Det}(H)=0$. Here one of the green curves passes very near to the SP; it is due to the special choice of $a_{0}$.

By the definition of the PES, Eq.(1), it is trivial that it is continuous and continuously differentiable to every order. Usually, also the location of the minimums, or the SPs, is 'stable' and changes continuously with the parameter $a_{s}$. The fact does not depend on the character of the parameters $a_{s}$ and the mean spacing $\tilde{a}_{o}$. They can be commensurable or incommensurable real numbers. In contrast, we have to deal with a whole number of atoms (it means the integer) which are sorted into a (may be other) whole number of wells of the on-site potential. An exception is a change of the character of the PES at all, like in an example below, in Subsection 5.2. The change here depends on the parameter $k$.

In Fig. 4 three NTs and the curves $\operatorname{Det}(H)=0$ (green) are included. The crossing of an NT with such a curve is a BBP point. Taking Example 1 and starting from the initial point $\left(x_{1}, x_{2}\right)=(2.0,6.0)$ the Barnes-algorithm [86] converges in very few iterations to the optimal $\operatorname{BBP}\left(x_{1}, x_{2}\right)=(2.09,6.46)$. It is $\mathbf{g}_{\text {opt }}=(0.69,0.35)$. The normalized optimal direction of the force is $\mathbf{l}_{o p t}=(0.89,0.45)$. The optimal amount of the force is $|\mathbf{g}|=0.77$ and the PES function is here $V=1.53$. Of course, the point falls in the manifold $\operatorname{Det}\left(H\left(x_{1}, x_{2}\right)\right)=0$ according to Fig.4. The optimal force is lower than the force at the $\mathrm{BBP}_{(1,1)}$ with 0.85 . The corresponding NT to direction $\mathbf{l}_{\text {opt }}$ is between $\mathrm{NT}_{(1,1)}$ and $\mathrm{NT}_{(1,0)}$.

At the BBP to the standard direction $(1,1)^{T}$ we still explain the tilted on-site potential with the external force $-0.85(1,1)^{T}$, in Fig. 5 . The tilting causes a coalescence of the upper minimum with the SP to a shoulder of the combined PES of string and on-site potential. 


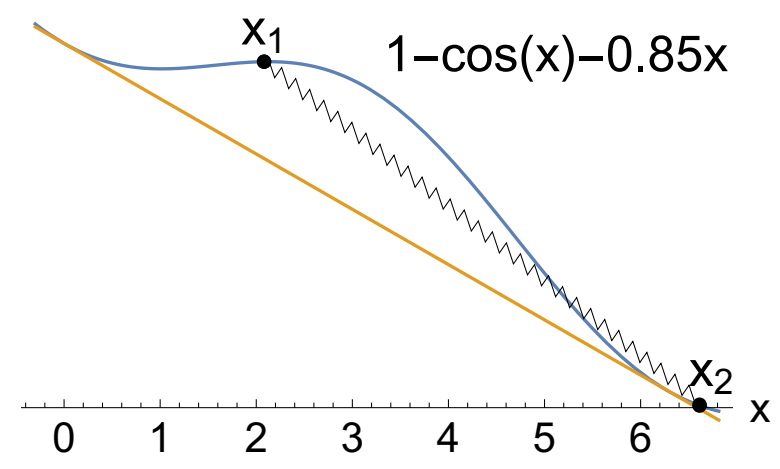

Figure 5. Schematic structure of the 'chain' with 2 atoms at the BBP, in relation to the tilted on-site potential. Compare Figs. 1 to 3. The orange line indicates the slope -0.85. The spring length is still 4.53 (against 4.19 of the pure spring). Note that the location of $x_{1}$ on the on-site potential is only for an explanation. Here one can 'see' that the structure is short before starting to slide downhill the shoulders of the effective PES consistently. The depinning starts.

\subsection{A 'Bifurcation' of the PES - Aubry Transformation}

If one treats another value of the parameter $k$, in comparison to Example 1, then some 'bifurcations' of the PES itself can emerge. This is sometimes named Aubrytransformation [34], or unfolding of a saddle-node equilibrium. First, for $k=0$, only the on-site potential remains. Here exists not only the diagonal valley like in Fig.1, along the former trough, but all of the double periodic minimums of the two cosine functions are possible. They form a $2 \mathrm{D}$ net of minimums. The atoms can both be in one well, or they can have all distances of $2 n \pi$ with all integers for $n$.

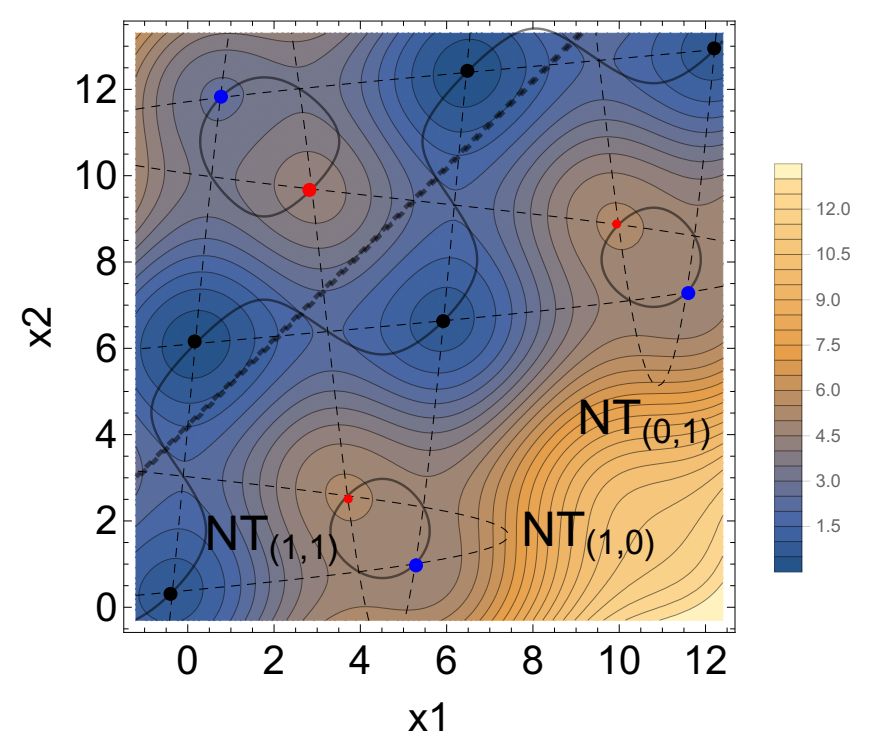

Figure 6. PES for a 'chain' with 2 atoms with locations $x_{1}$ and $x_{2}$ on one line. The relation parameter $k=0.05$ is very small. The minimums in the main valley are black bullets, but the intermediate side minimums are blue bullets. Red points are maximums, or SPs of index 2. The fat curve is a MEP through the main minimums. It is the $\mathrm{NT}_{(1,1)}$. The dashed NTs are the special ones to the coordinate directions. The fat double-dashed line is the ground line of the spring potential. 


\section{Example 2:}

Be $k>0$ but small $k \ll 1$, and $v=1$. We take $k=0.05$, and again $N=2, a_{o}=4 \pi / 3$ and $a_{s}=2 \pi$ like in Example 1. The potential of the spring allows the former central valley with the ground states. This valley never disappears for $k>0$, but here intermediates still emerge in 'second rows' left and right of the main valley, see Fig.6. It is a light blue strip of the PES 'above' the MEP, and a brown strip of the PES 'below' the MEP. Along the main valley a qualitative change has also happened. The minimums are different. There are global minimums near $(0,2 \pi)+\mathrm{n}(2 \pi, 2 \pi)$, n integer, and intermediate minimums near $(0,0)+\mathrm{n}(2 \pi, 2 \pi)$. Which kind of minimum emerges here depends on the parameter $a_{0}$ of the spring correspondingly. (If one additionally changes $a_{0}$ then this moves the double-dashed line in Fig. 6. It also changes the distances of the diverse minimums to this line.) The global minimums represent the former 'correct' pair $x_{1}-x_{2}$ with an elongated distance near $2 \pi$, but the intermediates represent 'anti-kinks' [90], where both atoms are in the same minimum valley of the on-site potential. Of course, their distance then is strongly shortened. It is possible because the spring constant is so low. A movement along the central valley now does not move the 'correct' pair, however, it goes on by a stepwise change between the 'correct' pair and the 'anti-kink' pair. The chain is unstable against pinning [34]. The corresponding SP is named the Peierl barrier here [34].

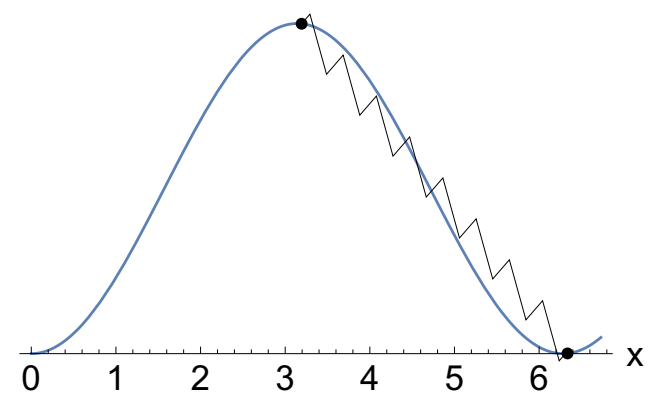

Figure 7. Schematic SP structure for the Peierls barrier of the two atoms on the ground line of Fig. 6, the fat $\mathrm{NT}_{(1,1)}$. The spring constant $k=0.05$ is very small, thus, the left atom is (labile) placed on the top of the on-site potential.

On the central MEP, the chain can now have different forms. In the global minimums, the chain looks like in Fig. 2. The intermediate minimums on the MEP along the double-dashed line look like in Fig. 3. But the spring is more extended in the first case, and more compressed in the second case. Both states are named 'pinned' [91]. However, now emerges a new form for the SP in between, see Fig. 7. If the 'chain' is at the SP structure then an infinitesimally small force moves down the corresponding atom from the top. This is named 'depinning' [91]. However, it needs a force before to come into this SP structure. Note that the 'main' NT through the main valley does not connect with the intermediate minimums of the second row, the blue bullets. They are crossed by isolated branches of the $\mathrm{NT}_{(1,1)}$. The minimums 'below' the main valley characterize a structure where the pair looks like in Fig.2 but with interchanged atoms (what is 'forbidden' for an ordered chain, from a theoretical point of view. However, the very smallness of $k$ does here not exclude such a structure.) The minimums 'above' the main valley depict pairs with a distance of $\approx 4 \pi$. But again like in example 1 , a further extension is forbidden by the spring. The new rows of stationary points, above and below the global zero line of the spring potential, are 
caused by the extreme smallness of the parameter $k$. However, the stationary points near the global zero line always stay there. For all forms of the chain from larger $k$ survive the minimums. May be new minimums emerge. However, to name the changes for $k \ll 1$ 'extreme discretization' [32] seems us not correct. No, if $k$ is too small then many, or all atoms can be packed into own well. The discretization can break down.

We emphasize that the step from example 1 to example 2 is a very natural possibility. The PES of example 2 is continuously differentiable, as well as the PES of example 1. The link between the two PESs of example 1 and example 2 is a PES where shoulders emerge. Beginning with the shoulder the new SP and the new minimum of example 2 develop. The structure of the transition is named a 'catastrophe' [94, 95]. Note that it is dependent on the parameter $k$. Here the critical value is $k_{c}=0.1038$ for a first bifurcation by an upper shoulder near point $(2.0,10.55)$. For a second $k_{c}=0.071$ the lower shoulders emerge near points $(4.5,1.85)$ and $(10.8,8.0)$. The rule is: the smaller the parameter $k$ is, the more minimums emerge of the $N$-dimensional net of the sinusoidal potential. An Aubry-like transition concerns a change of the model parameter. It is not a property of a fixed model of a given chain of atoms along Eq.(1) $[22]$.

\subsection{Example 3: Triatomic 'Chain' [59, 96]}

The case $N=3$ is treated with the same parameters like in Example 1. For the part of the PES between $x_{2}$ and $x_{3}$ holds an analogous picture like in Fig. 1 for $x_{1}$ and $x_{2}$. One could think that for the additive combination of both parts we get again an MEP with one $\mathrm{SP}$ in between [91]. However, $x_{2}$ is used in both parts. So the result is different. In

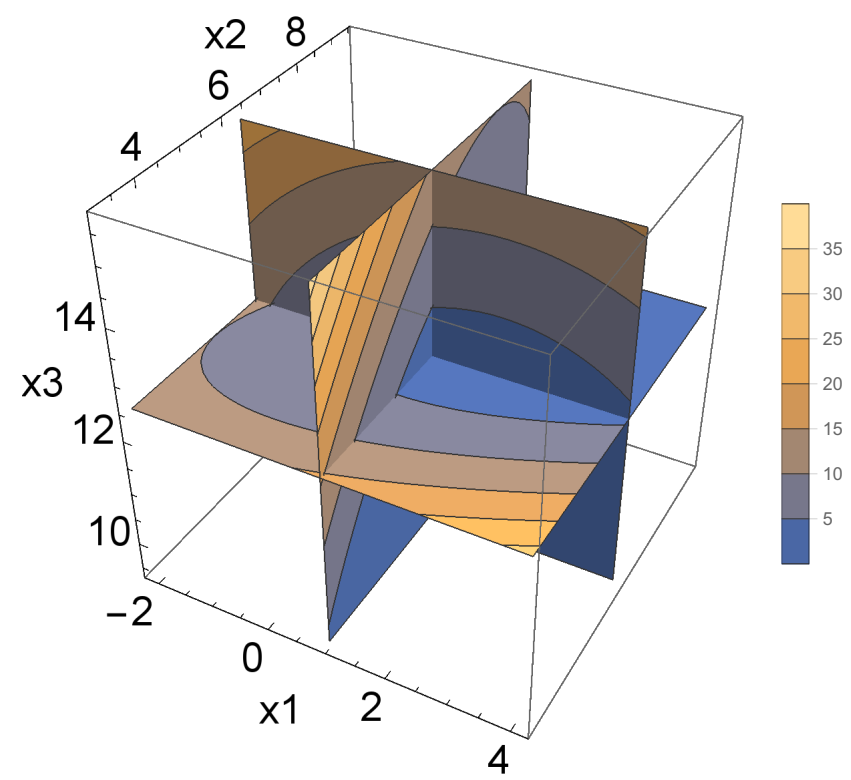

Figure 8. Spring potential of the 3-atomic 'chain' with parameters like in Example 1. Slices are drawn for the three different axes combinations.

Fig. 8 we show three projections of the spring potential. Three slices of the $4 \mathrm{D}$ surface over the 3D configuration space are given. In the planes, the typical pattern of a flat trough potential is replaced by curvilinear potential lines with a clear minimum. Of 


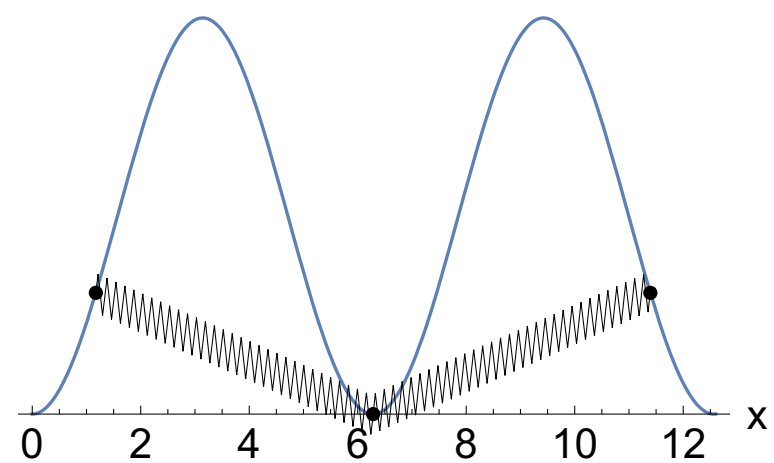

Figure 9. Schematic minimum of the 3-atomic 'chain' with parameters like in Example 1. This is a symmetric minimum structure. It is very stable. 3 atoms are placed in 3 wells of the on-site potential. Its energy is 2.074 .

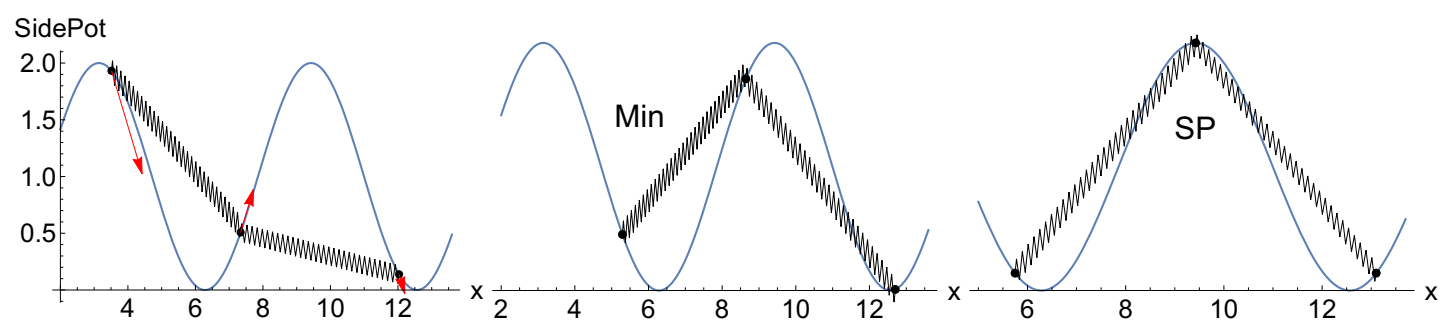

Figure 10. Asymmetric 'pre-SP', intermediate minimum of an anti-kink, and symmetric SP of the 3-atomic 'chain' with parameters of Example 3. They are calculated along the $\mathrm{NT}_{(1,1,1)}$ for $\mathrm{N}=3$, compare Fig.11. The energies are from left to right $2.77162,2.53051$, and 2.54181 . The red arrows at the left SP are the eigenvector to eigenvalue -0.35 along the SP valley. Of course, on the right hand side, there an equivalent intermediate minimum continues, and a further SP with mirror symmetry, the 'post-SP'.

course, a flat ground line survives in the case $x_{2}-x_{1}=x_{3}-x_{2}=a_{o}$, or $x_{2}=\frac{x_{1}+x_{3}}{2}$ and $x_{3}=x_{1}+2 a_{o}$. To localize the minimum, we apply a corresponding search program for NTs developed in Section 4.2. The minimum for the atoms is shown in Fig. 9. In this case, with a well-balanced parameter $k=1$ between string and on-site potential, a complicated new situation emerges. There are three SPs and two intermediates on the MEP from one global minimum to the next, drawn in Fig. 10. The atoms of the 3 -chain at diverse special points are given in Table 1 and their positions are included in Fig.11.

Table 1. Atoms of the 3-chain at stationary points

\begin{tabular}{ccccc}
\hline Node & $x_{1}$ & $x_{2}$ & $x_{3}$ & Energy \\
\hline Minimum & 1.173 & 6.283 & 11.394 & 2.07419 \\
pre-SP & 3.513 & 7.339 & 12.034 & 2.77162 \\
int-Min & 5.291 & 8.643 & 12.699 & 2.53051 \\
sym-SP & 5.747 & 9.425 & 13.103 & 2.54181 \\
int-Min & 6.15 & 10.207 & 13.558 & 2.53051 \\
post-SP & 6.816 & 11.525 & 15.385 & 2.77162 \\
Minimum & 7.456 & 12.566 & 17.677 & 2.07419 \\
\hline
\end{tabular}

Note that the asymmetric SPs, the 'pre-SP', and the 'post-SP', are higher in energy than the symmetric SP. But the asymmetric SPs have none of their atoms exactly on the top of the on-site potential, and to overcome these asymmetric SPs is here the main step of the Peierls barrier. This is the effort to move a single outer atom over the barrier of the on-site potential under the action of the spring force to form an 


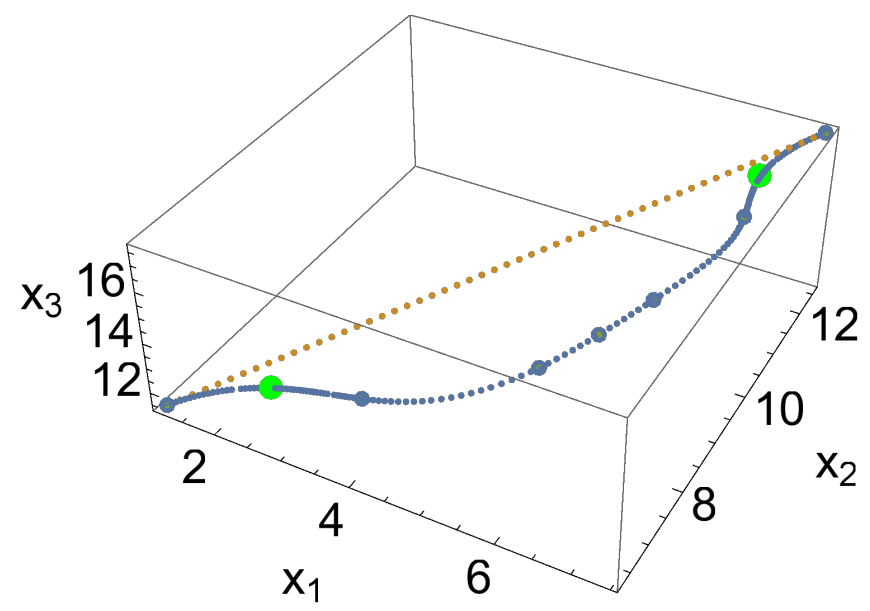

Figure 11. Calculated $\mathrm{NT}_{(1,1,1)}$ (blue with $L=153$ nodes) of the 3-atomic 'chain' with parameters of Example 3. The NT is drawn in the 3D coordinate space of the chain. The fat blue bullets are the stationary points of Table 1. The fat green bullets are the outer BBPs. The orange line is given for comparison. It is the straight line between the two global consecutive minimums with direction $(1,1,1)$.

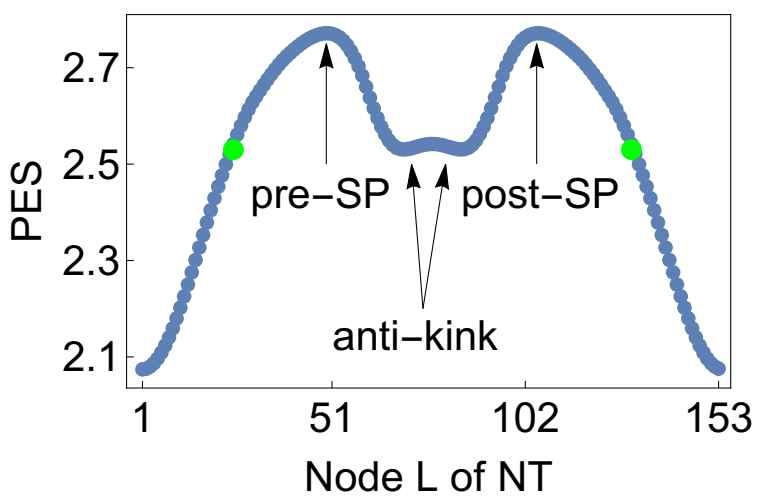

Figure 12. PES profile of the 3-atomic 'chain' over the used NT. The two highest SPs are on the left hand side the 'pre-SP', and on the right the 'post-SP'. The global minimums are at nodes 1 and 153 . The energy of the barrier is 0.698 .

anti-kink. It pushes two atoms in one potential well. It concerns $x_{1}$ at the 'pre-SP', or $x_{3}$ at the 'post-SP'. The profile of the PES over the $\mathrm{NT}_{(1,1,1)}$ is represented in Fig.12. Of course, such a profile over the NT can be used for a first guess of the diverse stationary points. (But sometimes the BBP is higher in energy than the next SP [65]. The NT can have a turning point. Such cases must be treated in more depth.) A pair of the chain forms an 'anti-kink' in the intermediate region [90]. The atom $x_{2}$ can easily interchange the partner jumping over the symmetric SP, with very little energy. Dynamically, one can imagine a 'vibrating anti-kink state' where atom $x_{2}$ oscillates over the symmetric SP [97].

The two outer BBPs are depicted by green bullets in Fig. 11. They are at $(2.32,6.97,12.0)$ with $\mathbf{g}=0.274(1,1,1)^{T}$, and at $(6.85,11.88,16.53)$ with $\mathbf{g}=$ $-0.274(1,1,1)^{T}$. Again, like for the case $N=2$, here the translation is not uniform from one global minimum to the next. The distances between the three atoms always change. The path is curvilinear, see Fig. 11. 


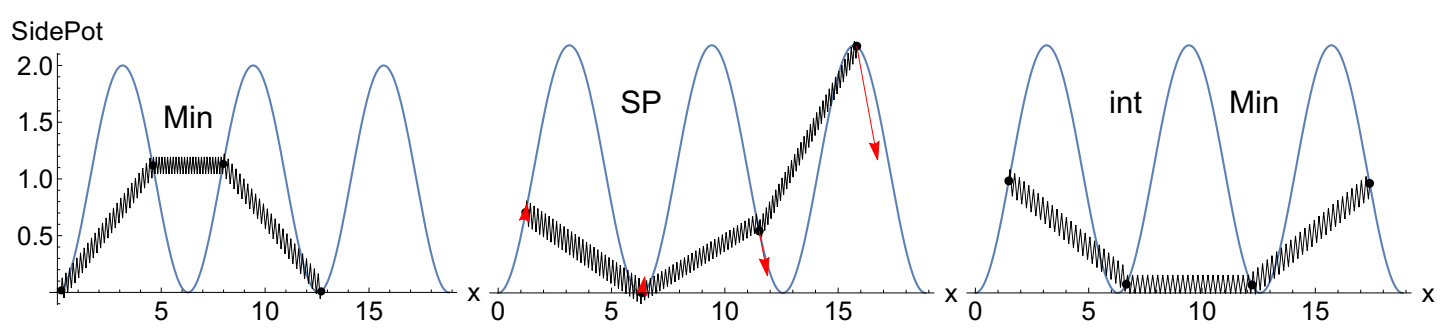

Figure 13. Schematic picture of the global minimum (left), SP, and intermediate minimum of the 4-atomic chain. The energies are $V=2.646,4.082$, and 3.84. The red arrows at the SP are the eigenvector to eigenvalue -0.387 along the SP valley. To the right hand side, a continuation with an equivalent further SP exists with mirror symmetry to this SP here, and then the next global minimum emerges.

\subsection{Example 4-atomic Chain [91, 92, 98]}

The case $N=4$ is treated with the same parameters like in Example 1. We find a global minimum, two SPs, and an intermediate minimum. The ground state has already a pair of atoms in one well of the on-site potential which cannot move as a pair to the next global minimum. To disconnect the pair, the first SP has mainly to concern atom $x_{3}$. First it has to overcome the top of the on-site potential, see Fig.13. It is in contrast to the former pre-SP in the case $N=3$ where atom $x_{1}$ is mainly included. The intermediate minimum, the right structure in Fig. 13, is a kink. Its four atoms are in four wells, but the global minimum has four atoms in three wells of the on-site potential. The chain is characterized by a rarefication of the 4 atoms.

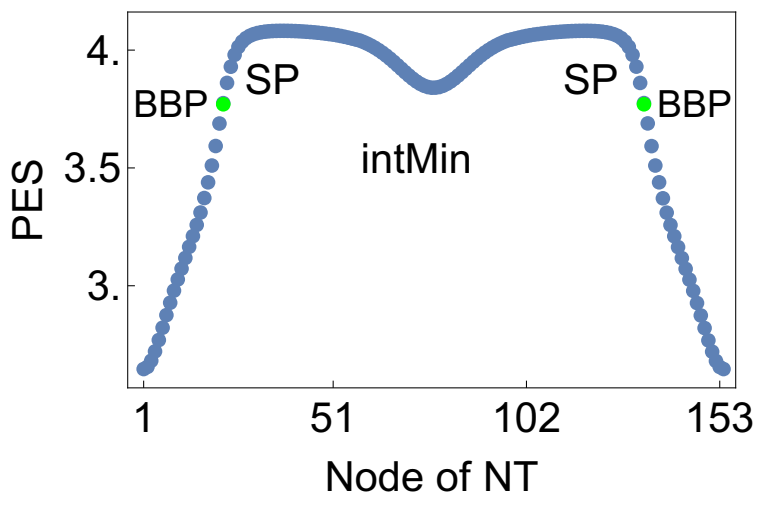

Figure 14. Energy profile between global minimum, two SPs, and a symmetric intermediate minimum of the 4 -atomic chain. The green bullets are the BBPs. The NT for the search goes along the direction $(1,1,1,1)^{T}$. The energy of the barrier is 1.44 .

The MEP is simpler than in the $N=3$-case. See Fig. 14 . The two outer BBPs have an energy of 3.77 , the left point is at $(1.244,4.718,9.423,14.357)$, and the right point is at $(3.139,8.074,13.811,17.284)$. They are depicted by green bullets in Fig. 14. The pathway which connects the global minimum over the SP, intermediate, next SP to the next global minimum, is an MEP. Besides the MEP, there exists a further (flat) SP in the region near $V=5.0$. It is of index 2 . Its coordinates are $(1.475,6.66,12.204,17.394)$. For an 'infinite' chain, the structure is declared as the usual SP [91]. Its eigenvalues are $(3.809,2.081,-0.277,-0.005)$. It is the symmetric version between the two asymmetric SPs of the MEP. However, this symmetry costs further energy. The NT used to find this $\mathrm{SP}_{2}$ was taken to the special direction $(1,0,0,-1)^{T}$. Other probed directions were 
not successful.

\subsection{Example 5-atomic Chain [34]}

The case $N=5$ is treated again with the same parameters like in Example 1. We find a global minimum, two SPs, and an intermediate minimum like in the Subsect. before. The symmetric ground state has the central atom $x_{3}$ on the top of the on-site potential. The case is discussed also in Refs. $[27,34,70,99]$. The symmetry breaks down if $k$ becomes too small. It is then a case like the 'bifurcation' of the PES in Subsect. 5.2. The interesting structures on the MEP are shown in Fig.15. Note that the other atoms are not at the ground zero of their wells of the on-site potential. However, this happens for atom $x_{3}$ at the intermediate. For the 5-atomic chain, as well, the global minimum contradicts an imagination that the atoms of the chain "reorganize towards lattice minima and avoid lattice maxima..." [34].

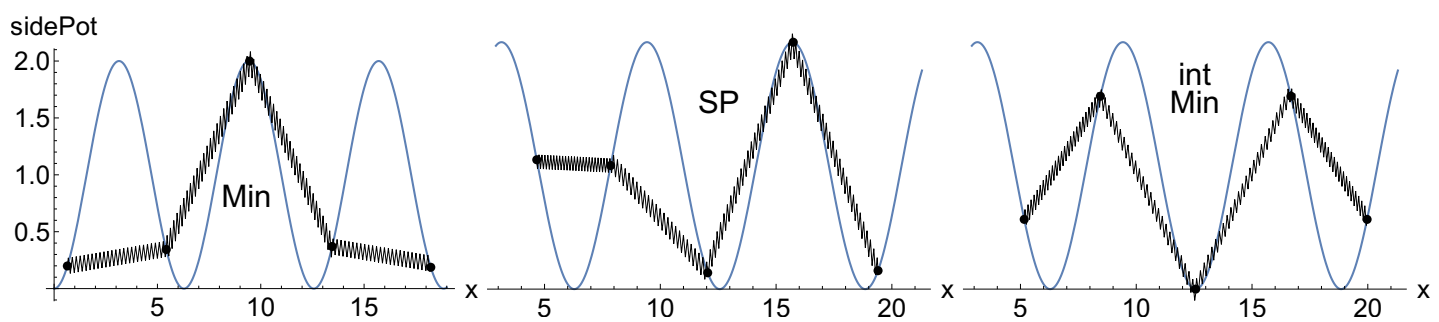

Figure 15. Schematic picture of the global minimum (left), SP, and intermediate minimum of the 5-atomic chain. The energies are $V=3.479,5.089$, and 5.07. On the right hand side, a continuation with an equivalent further SP exists with mirror symmetry to this SP here, and then the next global minimum emerges. A similar structure for the global minimum was reported elsewhere for an SP [92].

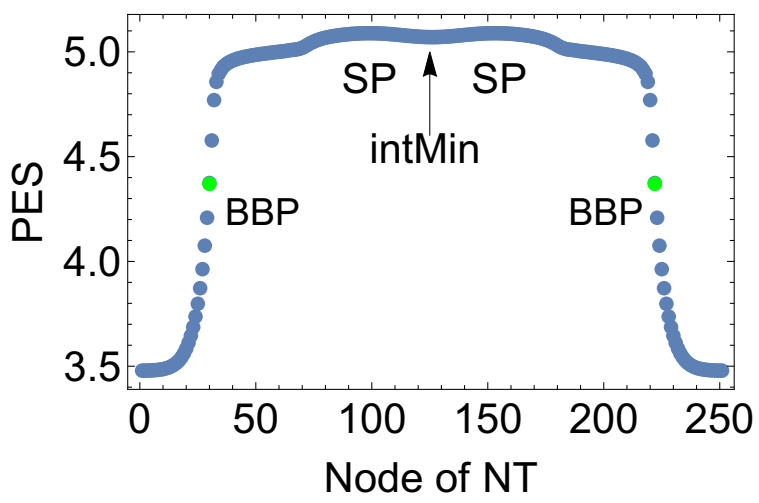

Figure 16. Energy profile between the global minimum, two SPs, and a symmetric intermediate minimum of the 5 -atomic chain. The green bullets are the BBPs. The NT for the search goes along the direction $(1,0,0,0,1)^{T}$. It is started from the intermediate minimum, it goes over the global minimum, and goes further to the next intermediate. Here it is depicted in the order of the former profiles. The energy of the barrier is 1.61 .

To get the MEP by an NT, we have to change the search direction from the standard one, $(1,1,1,1,1)^{T}$, to the 'push and pull' direction $(1,0,0,0,1)^{T}$. The NT to the former direction does not find a low-energy path over the PES. The MEP is still more flattened in the high region than in the $N=4$-case, see Fig. 16 . The intermediate nearly seems to disappear. It means that a state with the corresponding 
structure of the chain becomes improbable. If we want to move the chain, we have to do it in one large step which jumps over the whole high region. For the step, the two outer BBPs have an energy of 4.375. They are depicted by green bullets in Fig. 16 .

Again beside the main MEP, an SP of index 2 exits, see a picture in Subsect.7 below.

The energy of the barrier for $N=2$ is 1.837 . The barrier increases from $N=3$ with 0.698 to $N=4$ with 1.44 to $N=5$ with 1.66 , but decreases to $N=23$ with 1.35 [25]. The barriers of these cases are lower than the assumed $\approx 2$ units [29].

\subsection{Example 23-atomic Chain}

A chain of few tens of ions is studied elsewhere [8,72]. The chain we will be looking at is the case $N=23$, with the same parameters like in Example 1 . Thus we use $a_{s}=2 \pi$, $a_{0}=4 \pi / 3, v=k=1$ (misfit parameter $2 / 3$ ). One can find a minimum, as well as a low lying SP with the method described in Sect.4.2. The minimum is asymmetric, but the SP is symmetric, both shown in Fig. 17 left panel and center. The energies are $V(\min )=19.8242$ and $V\left(S P_{1}\right)=19.9644$. For the minimum is $\tilde{a}_{0}=4.12$, quite near to the natural $a_{0}=4.19$. One can mirror the structure of the minimum at $30 \pi$ and reverse
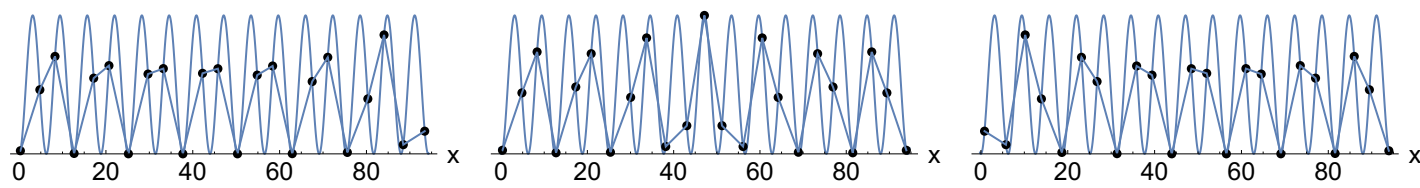

Figure 17. Schematic picture of the structures of the 'left' asymmetric minimum of the 23-atomic chain, the symmetric SP, and the 'right' asymmetric minimum. The energy is 19.8242 for the minimums, and 19.9644 in between.

the chain, to get the other asymmetric minimum with the same energy, compare Fig. 17 on the right hand side. The data of the structures are given in the SM, Sect.1.1. and 1.2. The 23 atoms of the chain are distributed to 16 wells of the on-site potential. It is a coverage of 1.44 atoms per well. If we imagine a movement of the chain from the 'left' minimum to the 'right' minimum, one has to move in a first step up to the SP the atoms $x_{15}, x_{18}, x_{21}$ into a new well but the $x_{12}$ to the top between two wells. On the other side downhill to the 'right' minimum, the $x_{12}$ is moved into its new well, and the atoms $x_{3}, x_{6}, x_{9}$ move into a new well. Note the triple numbering of the moving atoms, and note that the SP has only its central atom $x_{12}$ at a top of the on-site potential. So it appears like a stable structure.

There is an additional $\mathrm{SP}_{1}$ between the two minimums, for the structure see Fig. 18, and the data are in the SM, Sect. 1.4. Its energy is higher than the SP of Fig. 17, center. Over this SP leads a second path between the two global minimums.

Between the two SPs of index 1 an $\mathrm{SP}_{2}$ (of index 2) emerges, for the structure see Fig. 19, and for the data see the SM, Sect.1.5. The 23 atoms of the SP are to distribute in the 16 wells of the on-site potential. 3 atoms are at the top of the on-site potential, and further 4 atoms are near the top of the on-site potential. The high number of atoms being on the tops of the on-site potential corresponds well with the usual imagination of an SP. However, it is already a more instable structure being an SP of index 2. Its energy is 20.2139. For this SP of index two only one singular NT exists from the minimum to the $\mathrm{SP}_{2}$, caused by the index theorem [67]. One can find the singular NT by a variational search $[100,101]$. The two negative eigenvalues here 


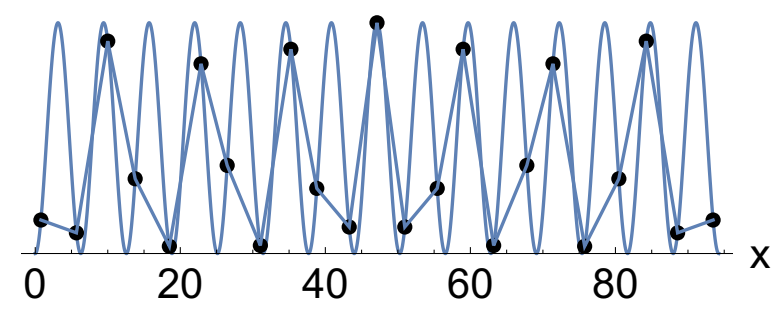

Figure 18. $\mathrm{SP}_{1}$ at 20.1993 units of energy; it is near in energy to the $\mathrm{SP}_{2}$ of Fig. 19.

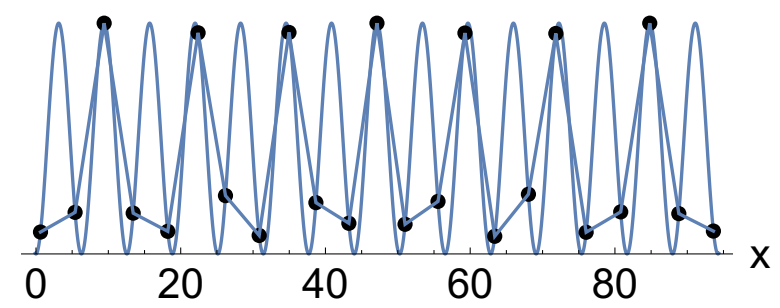

Figure 19. A low lying $\mathrm{SP}_{2}$ of index 2 of the 23-atomic chain. The energy is 20.2139 . It is symmetric and connects the two minimums over a singular NT, see SM, Sect. 1.5.

are -0.073 and -0.037 . Thus, the SP is quite flat. The next 3 positive eigenvalues are also quite small. The $\mathrm{SP}_{2}$ forms a small, flat plateau over the paths through the two lower SPs of index one.

On a much more complicated level, possible dynamic transitions between the two minimum structures along the double minimum valley of the MEP correspond to an anti-kink 'vibration' of atom $x_{12}$ like in example $N=3$. Additionally, on the right hand side of the MEP, the atoms $x_{3}, x_{6}$, and $x_{9}$ change their well, but on the left hand side of the MEP, the atoms $x_{15}, x_{18}$, and $x_{21}$ change the well. The energy difference, the barrier height of the lower $\mathrm{SP}_{1}$, is only 0.14 . However, it is not a movement of the structure along the axis at all. The distance between the two minimum structures is 1.665 in average between the atoms, only $\approx \pi / 2$. So to say, the structure would vibrate on its place, if it moves over the SP region, compare Fig. 17. This 'anti-kink' vibration is pinned into the on-site potential, or in other words, it is an immobile state. It is adapted to another concept, this of a breather being a "spatially localized and time periodic solution" $[46,102]$. And here we do not have the case of a 'propagating breather' [103]. - But here we do not further discuss dynamic effects.

\section{The Twist Map representation}

\subsection{The case $N=2$}

Often celebrated is the representation of the stationary structure of the FK model by a standard map $[24,104,105]$. We test it for the two-atoms 'chain'. The gradient for $N=2$ is

$$
\begin{aligned}
& g_{1}=2 \pi / a_{s} \sin \left(2 \pi x_{1} / a_{s}\right)-k\left[x_{2}-x_{1}-a_{o}\right] \\
& g_{2}=2 \pi / a_{s} \sin \left(2 \pi x_{2} / a_{s}\right)+k\left[x_{2}-x_{1}-a_{o}\right] .
\end{aligned}
$$


The system of equations, $g_{1}=0, g_{2}=0$, is solved by stationary solutions of the FK chain. The system can be reformulated by

$$
\begin{aligned}
& x_{2}=2 \pi /\left(k a_{s}\right) \sin \left(2 \pi x_{1} / a_{s}\right)+\left[x_{1}+a_{o}\right] \\
& x_{1}=2 \pi /\left(k a_{s}\right) \sin \left(2 \pi x_{2} / a_{s}\right)+\left[x_{2}-a_{o}\right] .
\end{aligned}
$$

Of course, a single step in Eq.(23) which presents the value of $x_{2}$ to a given $x_{1}$, is not enough for the calculation of the next stationary point. It only fulfills the Eq.(21) for $g_{1}=0$. One has to do the iteration cycles of Eqs.(24), and (23), up to convergence.

We do not need an iteration since we start with the correct value of the component $x_{1}$ at a stationary point. Then $x_{2}$ of the equilibrium chain results; and the iteration is finished because all next iterations are also fulfilled. However, if one starts with values outside a stationary point, one may get slowly converging iterations, to any stationary point. An iteration by Mathematica with the parameters of example 1 is shown in scheme 1.

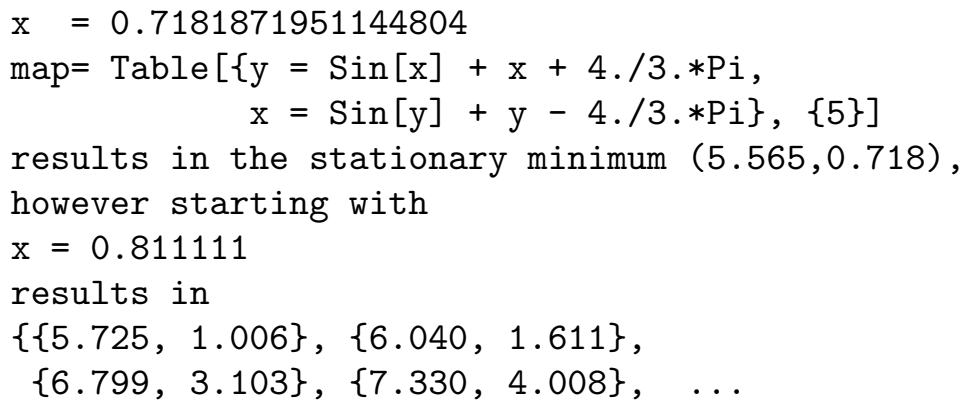

Scheme 1: Iteration of the case $N=2$, see Fig. 20.

The iteration is very slowly convergent; after 150 iterations it is exact for three digits after the point, however at the other stationary structure, the SP at (4.689, 7.878). It is known that gradient methods work poorly for minimizations [106]. The twist map method is a further example for this statement.

However, we comment here that the method has a strong connection to Newton trajectories. Eq.(21) with $g_{1}=0$ means that we search a point on a curve where the first component of the gradient is zero, thus the second component is still free. This means we search a point on an $\mathrm{NT}_{2}$ with direction of the force $\mathbf{l}_{2}=(0,1)$, see Fig. 20. Eq.(22) with $g_{2}=0$ means that we search a point on a curve where the second component of the gradient is zero, thus the first component is again free. It means we search a point on an $\mathrm{NT}_{1}$ with the direction of the force $\mathbf{l}_{1}=(1,0)$. And so on iteratively. The iteration of this twist map is shown in Fig.20. The iteration jumps between the two special NTs to the coordinate directions. Only after the convergence, the complete system $g_{1}=0, g_{2}=0$ is fulfilled, and not only single equations; and we are at a stationary point. The zigzagging between the NTs explains the slow convergence speed. The deficiency is especially strong because here both NTs go in an asymptotic kind against the SP. - In the special case of the misfit parameter $2 / 3$ which is used here.

A further property of the iteration ansatz of system $(23),(24)$ is that the search goes 'uphill', thus to the next SP, a metastable state, not to the next minimum. With the 


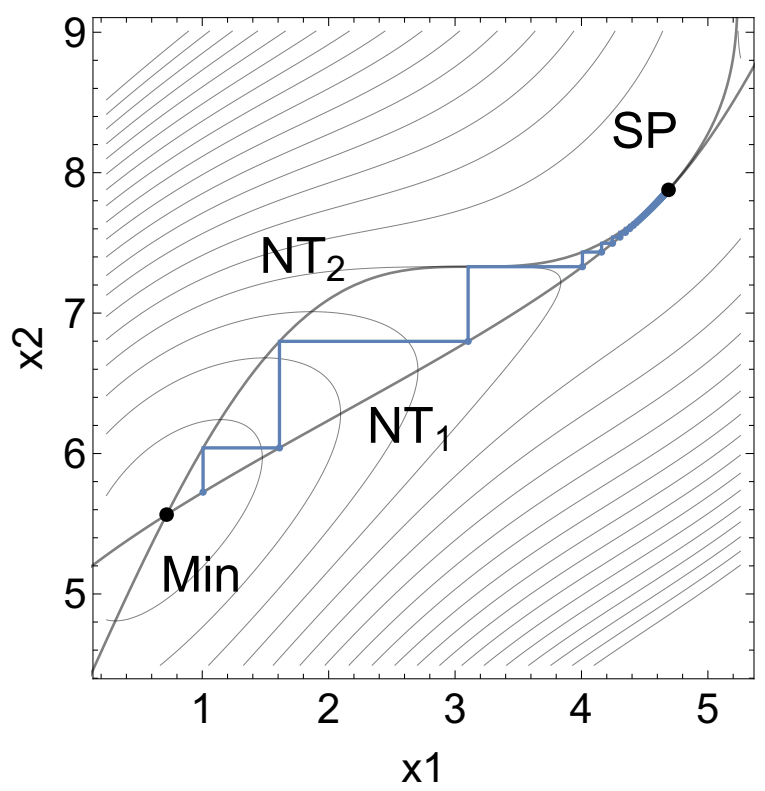

Figure 20. Iteration of a twist map for the 2-atoms 'chain' of Section 5.1. Blue steps start near the Min and jump between the two NTs in the coordinate directions. The iteration converges to the SP, however, very slowly.

knowledge of the former kind of iteration, we can turn the direction by an analogous 'mixed' ansatz

$$
\begin{aligned}
& x_{1}=-2 \pi /\left(k a_{s}\right) \sin \left(2 \pi x_{1} / a_{s}\right)+\left[x_{2}-a_{o}\right] \\
& x_{2}=-2 \pi /\left(k a_{s}\right) \sin \left(2 \pi x_{2} / a_{s}\right)+\left[x_{1}+a_{o}\right] .
\end{aligned}
$$

Starting again at $x_{1}=0.811111$, and $x_{2}=5.564998$, the iteration finds the nearby minimum in approximately 30 steps with a much higher accuracy. Now the staircase goes downhill. However, by the zigzagging between the two NTs, the convergence is not good.

\subsection{The cases $N>2$}

The system of zero gradient equations for more than two atoms has the following general line [24]

$$
2 x_{i}-x_{i-1}-x_{i+1}+\frac{2 \pi}{k a_{s}} \sin \left(\frac{2 \pi x_{i}}{a_{s}}\right)=0
$$

for $2 \leq i \leq N-1$. For $i=1$ the Eq.(3) counts, and for $i=N$ the Eq.(4). Given any initial value for $x_{1}$ we can fulfill the first equation, and then one line after the other in Eqs.(27). However, if the initial $x_{1}$ is not the component of a stationary structure of the chain, (which we cannot know from the outset) then the calculation will lead to a contradiction. The equation for $i=(N-1)$ of Eqs.(27) gives $x_{N}$ which usually will not be the same value in comparison to the one which the last equation, Eq.(4) for $N$, prescribes. This means we do not have a stationary structure, see the remark above on the case $N=2$. Now, an iteration of the map in the case of $N>2$ atoms 
does not work for more than one cycle, because $x_{N}$ and $x_{1}$ are not connected by a direct spring potential, like in ansatz (21) and (22) in the case of $N=2$.

The missing link is said to be by-passed in the case of an 'infinite' chain by doing the twist map on a torus 'infinity often'. What we question in general. Any arbitrary initial $x_{1}$ is to set. Arbitrary because we do not know the correct $x_{1}$ at the beginning. Some workers also continue Eqs.(27) below to $-\infty$ [70]. By this step the special role of an $x_{1}$ disappears. One has a free floating chain, without its physical context. Next, it loses the influence of the parameter $a_{o}$ but produces a random spacing $\tilde{a}_{o}$. We object the theorem that to every $\tilde{a}_{o}$ a minimum chain of the FK model (1) exits [40]. A still more serious problem is that if one has arrived the 'infinite' atom of the chain, ' $x_{\infty}$ ', one would have done only the first step of the iteration, and one is not at a stationary solution of the FK model (1). How to connect the ' $x_{\infty}$ ' with the corresponding next $x_{1}$ for an iteration, that has not been reported in Ref.[24], or that is only tried by a 'try and error' calculation [32]. We guess that a correct iteration was never done. The main fallacy in this kind of thinking is that the contradiction of the $(N-1)$ th equation for $x_{N}$ is moved to infinity; and the users assume that the contradiction disappears there.

There are other statements that there exists a periodicity for a large $N$. It is, however, also open which $N$ is concerned. Of course, one can put $x_{1}=x_{N}$ modulo $a_{s}$ with any $N$ for the next iteration step, see Eq.(13). Then in the end one will have an 'infinite', but periodic chain of the random, but fixed periodicity, $N$, after convergence.

In contrast, in the finite chain, however, we can make a symmetry assumption for the free ends

$$
x_{1}=2 C_{m p}-x_{N}
$$

where $C_{m p}$ is the midpoint of the chain, $x_{(N-1) / 2}$ for $N$ odd, or $\left(x_{(N+2) / 2}+x_{N / 2}\right) / 2$ for $N$ even. Of course, such an ansatz can only work if the chain is symmetrically embedded in the on-site potential, indeed, see below subsection 7. Asymmetric stationary points like the Pre-SP in the $N=3$ case above or the global minimums of the $N=23$ case cannot be detected by the method. With a symmetry assumption the iteration can go on up to convergence. Then we have a 'turn around zigzagging' using all the $N$ directional NTs to the $N$ coordinates. An example is the case $N=3$ where after 10 iterations the global minimum structure is found, shown in Fig. 9 and Table 1.

Some workers propose fixed-end boundary conditions [32], name it $x_{0}=\mathrm{b}_{0}$ and $x_{N+1}=\mathrm{b}_{0}+M a_{s}$ with an appropriate integer $M$. Then $\tilde{a}_{0}$ is fixed to $\left(x_{N+1}-x_{0}\right) /(N+$ $1)=M a_{s} /(N+1)$. The setting changes the PES Eq.(1) to

$$
V_{b}(\mathbf{x})=\sum_{i=1}^{N}\left[1-\cos \left(\frac{2 \pi x_{i}}{a_{s}}\right)\right]+\sum_{i=0}^{N} \frac{k}{2}\left[x_{i+1}-x_{i}-a_{o}\right]^{2} .
$$

The general gradient component, Eq.(2), is not changed but it holds for $i=1, . ., N$. However, if we use the constancy of $x_{0}$ and $x_{N+1}$, we can separate the two boundary 
equations by

$$
g_{1}=2 \pi / a_{s} \sin \left(2 \pi x_{1} / a_{s}\right)+k\left[2 x_{1}-x_{2}-b_{0}\right]
$$

and

$$
g_{N}=2 \pi / a_{s} \sin \left(2 \pi x_{N} / a_{s}\right)+k\left[2 x_{N}-x_{N-1}-b_{0}-M a_{s}\right]
$$

In comparison to the original gradient, Eqs.(2) to (4), we have to state that setting both gradients to zero is quite the same problem for the variables $x_{i}$ for $i=1, \ldots, N$. Especially if we use $x_{1}-b_{0}=a_{0}$ and $x_{N}-b_{0}-M a_{s}=a_{0}$, we get identical problems. The authors of Ref.[32] skate around by writing: ... an initial value "is unspecific, i.e. it can take any value which leads to the given $x_{N+1}$ " being $b_{0}+M a_{s}$ in Eq.(31). Our comment: this 'unspecificity' is exactly the problem. However, since the boundary points are symmetric, one could here use Eq. (28) as well for the initial value of the next iteration step.

\section{Thoughts on the symmetry of an FK solution}

Eq. (27) is derived from Eq.(2) with the condition $g_{i}(\mathbf{x})=0$ for $i=2, \ldots, N-1$. We now assume we have a stationary structure of the FK model. Eq. (27) can be rewritten

$$
\Delta x_{i}=\frac{2 \pi}{k a_{s}} \sin \left(\frac{2 \pi x_{i}}{a_{s}}\right)+\Delta x_{i-1}
$$

for $i=2, \ldots, N-1$ where $\Delta x_{j}=x_{j+1}-x_{j}$. Thus it is $\Delta x_{i}=f\left(x_{i}, \Delta x_{i-1}\right)$ for $i=2, \ldots, N-1$. For the case $i=1$ the equation takes the form from Eq.(3)

$$
\Delta x_{1}=\frac{2 \pi}{k a_{s}} \sin \left(\frac{2 \pi x_{1}}{a_{s}}\right)+a_{0}
$$

whereas for $i=N$ the expression is from Eq.(4)

$$
\Delta x_{N-1}=-\frac{2 \pi}{k a_{s}} \sin \left(\frac{2 \pi x_{N}}{a_{s}}\right)+a_{0} .
$$

Substituting the expression for $\Delta x_{1}$ into the expression for $\Delta x_{2}$ and so on we obtain

$$
\Delta x_{i}=\frac{2 \pi}{k a_{s}} \sum_{k=1}^{i} \sin \left(\frac{2 \pi x_{k}}{a_{s}}\right)+a_{0},
$$

for $i=1, \ldots, N-1$. Finally, if we substitute the last expression in the equation for $i=N-1$ then we have after some trivial rearrangements

$$
0=\sum_{j=1}^{N} \sin \left(\frac{2 \pi x_{j}}{a_{s}}\right)=\sum_{j=1}^{N} \sin \left(\xi_{j}\right) \quad \text { with } \quad \xi_{j}=\frac{2 \pi x_{j}}{a_{s}} .
$$

If $N$ is an even number, a skew-symmetric solution of Eq.(33) is possible by $\sin \left(\xi_{1}\right)=-\sin \left(\xi_{N}\right), \sin \left(\xi_{2}\right)=-\sin \left(\xi_{N-1}\right), \ldots, \sin \left(\xi_{N / 2}\right)=-\sin \left(\xi_{N / 2+1}\right)$ while if $N$ is an 
odd number a skew-symmetric solution is possible by $\sin \left(\xi_{1}\right)=-\sin \left(\xi_{N}\right), \sin \left(\xi_{2}\right)=-$ $\sin \left(\xi_{N-1}\right), \ldots, \sin \left(\xi_{(N+1) / 2-1}\right)=-\sin \left(\xi_{(N+1) / 2+1}\right)$, and $\sin \left(\xi_{(N+1) / 2}\right)=0$. Consequently, if $\sin \left(\xi_{i}\right)=-\sin \left(\xi_{k}\right)$ then holds $\cos \left(\xi_{i}\right)=\cos \left(\xi_{k}\right)$ because the sine function is at $\pi$ skew symmetric, but the cosine function is here symmetric. Clearly such solutions are confined to the symmetric case. However, asymmetric solutions of Eq.(33) are also possible. But Eq. (33) is an interesting result by itself.

Conclusion: The sum of all sine terms of the atom positions in the FK model is zero at an equilibrium structure, compare the Example in Fig. 21.

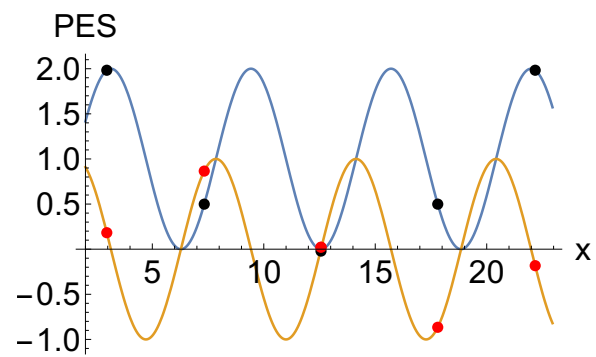

Figure 21. Schematic picture of an SP of index 2 of the 5-atomic chain. The energy is 6.097. A similar structure is reported elsewhere [70] but not for an SP of index 2. Compare the sine function overlay in orange. Blue is the usual $(1-\cos (x))$ profile. The atoms (in black) are artificially set on the $(1-\cos )$ function. They are (in red) put by an orthogonal projection onto the sine curve. The skew-symmetry for the sine terms is fulfilled. This means in the contrary direction that the structure is symmetric on the on-site potential profile, $(1-\cos (x))$.

Last, we have derived the energy of the FK model (1) at a stationary point $\mathbf{x}_{E Q}$ using Eq.(32). The expression is

$$
V\left(\mathbf{x}_{E Q}\right)=v \mathbf{1}_{N}^{T} \mathbf{c}\left(\mathbf{x}_{E Q}\right)+\frac{2}{k}\left(\frac{\pi}{a_{s}}\right)^{2} \sum_{i=1}^{N-1}\left(\mathbf{1}_{i}^{T} \mathbf{s}\left(\mathbf{x}_{i}\right)\right)^{2}
$$

where $\mathbf{1}_{i}^{T}=\left(1_{1}, \ldots, 1_{i}\right), \quad \mathbf{c}^{T}(\mathbf{x})=\left(1-\cos \left(\frac{2 \pi x_{1}}{a_{s}}\right), \ldots, 1-\cos \left(\frac{2 \pi x_{N}}{a_{s}}\right)\right)$ and

$$
\mathbf{s}^{T}\left(\mathbf{x}_{i}\right)=\left(\sin \left(\frac{2 \pi x_{1}}{a_{s}}\right), \ldots, \sin \left(\frac{2 \pi x_{i}}{a_{s}}\right)\right)
$$

\section{Conclusion}

Recently, experiments are done with laser-cooled and trapped ions for insights into friction processes $[34,59,72,107]$. The systems try to emulate the FK model (1) for a small number $N$, where the chain of the interesting particles slides under an external force over the fixed rigid on-site potential. The FK model (1) is not realistic in every detail. But it is sufficiently complex for tests of basic concepts [108]. Thus any theoretical work to understand such experiments better is useful.

In the past, there were different experiences. The competitive interaction of the different potentials in the FK model (1) "can make the determination of the ground state far from trivial ..." [12]. In contrast to the remark, we demonstrate in this paper 
that the stationary states like minimums and SPs of any index of the PES of the finite FK model (1) can be gotten by NTs (or any other minimization method). It is not problematic if an equilibrium state is asymmetric, and it is independent on the relation of the parts of the misfit parameter, the periodicities, $a_{s}$ and $a_{0}$. May they be rational or irrational, commensurable or incommensurable. We note that the application of the twist map may have concealed the existence of asymmetric equilibrium structures.

The PES (1) is directly given in the FK model. We can execute all calculations of NTs which determine the diverse properties of the model. The accuracy can be high. For comparison and confirmation we controlled the results of NT calculations by usual minimization procedures of the Mathematica program system because the PES of the FK model (1) is a usual surface where the known minimization procedures work.

NTs are especially appropriate to the driven FK model by a tilting force. By the calculation of a barrier breakdown point (BBP) on the NT of the driving force direction we get the depinning transition, and the gradient there is the critical force. The critical phenomenon is reducible to a saddle-node transition [70], or a shoulder on the effective PES. All that are static properties of the effective PES.

We observed kink or anti-kink relations in the different models. An interesting aspect of the medium example with $N=23$ atoms is that usually not single atoms form an anti-kink. No, groups, like sets of triplets are involved in steps along the NT from a minimum to an SP, and vice versa. Where two atoms of the triplet stay in their well but the third atom moves over the top of the on-site potential. Of course, this observation depends on the misfit parameter of $2 / 3$ used here.

NTs allow us to drive they in very different directions over the PES. The standard direction $(1, \ldots, 1)^{T}$ is physically well understandable. Our used directions in the higher dimensional cases, beginning with $N=4$, often deviate from the standard direction. The corresponding valleys on the corrugated PES, which may be also very curvilinear, are better to follow by NTs which are adapted to the situation. So we work often with a kind of 'abstract' tilting which concerns only parts of the chain with different weights, especially the simple 'push and pull'-direction $(1,0, \ldots, 0,1)^{T}$. However, so the individual peaks and valleys of the PES open, and so optimal forces are to get; and may be experimental workers can construct an experiment where such optimal forces are applied to the chain. With a lower load than it is necessary for a full movement of the chain in standard direction.

\section{Disclosure statement}

No potential conflict of interest was reported by the authors.

\section{Supplementary materials}

The authors confirm that the data supporting the findings of this study are available within the article and its supplementary materials. 


\section{Funding}

We acknowledge the financial support from the Spanish Ministerio de Economía y Competitividad, Project: CTQ2016-76423-P, and from the Generalitat de Catalunya, Departament d'Empresa i Coneixement, Project: 2017 SGR 348.

\section{References}

[1] O.M. Braun and Y.S. Kivshar, Phys. Rep. 306, 1-108 (1998).

[2] J. Tekić and P. Mali, The ac driven Frenkel-Kontorova model (University of Novi Sad, Novi Sad, 2015).

[3] O. Braun and A. Naumovets, Surf. Sci. Rep. 60, 79-158 (2006).

[4] A. Vanossi and O.M. Braun, J. Phys. Condens. Matter 19, 305017 (2007).

[5] C. Wang and Y. Yang, Commun. Theor. Phys. (Beijing, China) 54, 112 - 116 (2010).

[6] G. Grüner, Rev. Mod. Phys. 60, 1129-1181 (1988).

[7] R. Thorne, Phys. Today 1996, 42-47 (1996).

[8] I. Garcia-Mata, O.V. Zhirov and D.L. Shepelyansky, Eur. Phys. J. D 41, 325-330 (2007).

[9] J.P. Pouget, C. R. Physique 17, 332-356 (2016).

[10] O. Braun, T. Dauxois, M.V. Paliy and M. Peyrard, Phys. Rev. B 54, 321 (1996).

[11] E.G. Ekomasov, R.R. Murtazin, O.B. Bogomazova and A.M. Gumerov, J. Magnetism Magnetic Materials 339, 133-137 (2013).

[12] L. Trallori, Phys. Rev. B 57, 5923 (1998).

[13] W. Que and M.B. Walker, Phys. Rev. B 46, 14772 (1992).

[14] R.C. Dinsmore III, M.H. Bae and A. Bezryadin, Appl. Phys. Lett. 93, 192505 (2008).

[15] M.H. Bae, R.C. Dinsmore III, T. Aref, M. Brenner and A. Bezryadin, Nano Lett. 9, 1889-1896 (2009).

[16] R. Besseling, R. Niggebrugge and P.H. Kes, Phys. Rev. Lett. 82, 3144-3147 (1999).

[17] N. Kokubo, R. Besseling, V.M. Vinokur and P.H. Kes, Phys. Rev. Lett. 88, 247004 (2002).

[18] O. Iaroshenko, V. Rybalko, V.M. Vinokur and L. Berlyand, Sci. Rep. 3, 1758 (2013).

[19] O.V. Zhirov, G. Casati and D.L. Shepelyansky, Phys. Rev. E 65, 026220 (2002).

[20] S. Watanabe, H.S.J. van der Zant, S.H. Strogatz and T.P. Orlando, Physica D 97, 429 470 (1996).

[21] A.V. Ustinov, M. Cirillo and B.A. Malomed, Phys. Rev. B 47, 8357-8360 (1993).

[22] A. Vanossi, N. Manini, M. Urbakh and S. Zapperi, Rev. Mod. Phys 85, 529-552 (2013).

[23] N. Manini, O.M. Braun, E. Tosatti, R. Guerra and A. Vanossi, J. Phys. 28, 134006 (2016).

[24] S. Aubry, Physica 7D, 240-258 (1983).

[25] W. Quapp and J.M. Bofill, submitted (2018).

[26] W. Quapp, J. Theoret. Comput. Chem. 2, 385-418 (2003).

[27] S.R. Sharma, B. Bergersen and B. Joos, Phys. Rev. B 29, 6335 - 6340 (1984).

[28] Y. Braiman, J. Baumgarten, J. Jortner and J. Klafter, Phys. Rev. Lett. 65, 2398-2401 (1990).

[29] S.L. Shumway and J.P. Sethna, Phys. Rev. Lett. 67, 995-998 (1991).

[30] C. Baesens and R.S. MacKay, Nonlinearity 11, 949-964 (1998).

[31] T. Strunz and F.J. Elmer, Phys. Rev. E 58, 1601-1611 (1998).

[32] N. Theodorakopoulos, M. Peyrard and R.S. MacKay, Phys. Rev. Lett. 93, 258101 (2004).

[33] I.D. Mikheikin, M.Y. Kuznetsov, E.V. Makhonina and V.S. Pervov, J. Mater. Synth. Process. 10, 53-60 (2002).

[34] A. Bylinskii, D. Gangloff, I. Counts and V. Vuletić, Nature Materials 15, 717-721 (2016).

[35] S.N. Coppersmith, Phys. Rev. A 36, 3375-3382 (1987).

[36] B. Hu and J.Y. Zhu, Phys. Rev. E 65, 016202 (2001). 
[37] A. Patrykiejew and S. Sokolowski, Cond. Mat. Phys. 17, 43601:1-14 (2014).

[38] P. Bak, Rep. Prog. Phys. 45, 587 (1982).

[39] A. Xu, G. Wang, S. Chen and B. Hu, Phys. Rev. B 58, 721 (1998-II).

[40] S. Aubry and P.L. Daeron, Physica 7D, 240-258 (1983).

[41] W. Chou and R.B. Griffiths, Phys. Rev. B 34, 6219-6234 (1986).

[42] R.B. Griffiths and W. Chou, Phys. Rev. Lett. 56, 1929 (1986).

[43] C. Baesens and R.S. MacKay, Nonlinearity 17, 567-580 (2004).

[44] Z. Zheng, B. Hu and G. Hu, Phys. Rev. B 58, 5453-5461 (1998).

[45] O.M. Braun, A.R. Bishop and J. Röder, Phys. Rev. Lett. 79 (19), 3692-3695 (1997).

[46] O.M. Braun, B. Hu and A. Zeltser, Phys. Rev. E 62 (3), 4235-4246 (2000).

[47] O.M. Braun, H. Zhang, B. Hu and J. Tekic, Phys. Rev. E 67, 06602 (2003).

[48] A.B. Kolton, D. Dominguez and N. Gronbech-Jensen, Phys. Rev. Lett. 86, 4112 (2001).

[49] S. Slijepčević, Chaos 25, 083108 (2015).

[50] I. Sokolović, P. Mali, J. Odavić, S. Radosevic, S.Y. Medvedeva, A.E. Botha, Y.M. Shukrinov and J. Tekic, Phys. Rev. E 96, 022210 (2017).

[51] J. Odavic, P. Malik, J. Tekic, M. Pantic and M. Pavkov-Hrvojevic, Commun. Nonlinear Sci. Numer. Simulat. 47, 100-108 (2017).

[52] H. Li and S. Liu, Discr. Dyn. Nat. Soc. 47, 7081804 (2018).

[53] O. Braun, T. Dauxois, M. Paliy, M. Peyrard and B. Hu, Physica D 123, 357-367 (1998).

[54] M. Hirano and K. Shinjo, Phys. Rev. B 41, 11837 (1990).

[55] A. Socoliuc, R. Bennewitz, E. Gnecco and E. Meyer, Phys. Rev. Lett. 92, 134301 (2004).

[56] M. Dienwiebel, G.S. Verhoeven, N. Pradeep, J.W.M. Frenken, J.A. Heimberg and H.W. Zandbergen, Phys. Rev. Lett. 92, 126101 (2004).

[57] E. Gnecco, S. Maier and E. Meyer, J. Phys.: Cond. Mat. 20, 354004 (2008).

[58] E. Meyer and E. Gnecco, Friction 2, 106-113 (2014).

[59] A. Bylinskii, D. Gangloff and V. Vuletić, Sci. 348, 1115-1118 (2015).

[60] F. Axel and S. Aubry, J. Phys. A 20, 487 (1987).

[61] W. Quapp, M. Hirsch, O. Imig and D. Heidrich, J. Comput. Chem. 19, 1087-1100 (1998).

[62] W. Quapp, M. Hirsch and D. Heidrich, Theor. Chem. Acc. 100 (5/6), 285-299 (1998).

[63] J.M. Bofill and J.M. Anglada, Theor. Chem. Acc. 105, 463-472 (2001).

[64] R. Crehuet, J.M. Bofill and J.M. Anglada, Theor. Chem. Acc. 107, 130-139 (2002).

[65] W. Quapp and J.M. Bofill, Theoret. Chem. Acc. 135 (4), 113 (2016).

[66] W. Quapp, J.M. Bofill and J. Ribas-Ariño, J.Phys.Chem.A 121, 2820-2838 (2017).

[67] M. Hirsch and W. Quapp, J. Molec. Struct., THEOCHEM 683 (1-3), 1-13 (2004).

[68] B. Peters, A. Heyden, A.T. Bell and A. Chakraborty, J.Chem.Phys. 120, $7877-7886$ (2004).

[69] W. Quapp, J.Chem.Phys. 122, 174106 (2005).

[70] L.M. Floria and J.J. Mazo, Adv. Phys. 45, 505-598 (1996).

[71] S. Slijepčević, Nonlinearity 26, 2051-2079 (2013).

[72] J. Kiethe, R. Nigmatullin, D. Kalincev, T. Schmirander and T.E. Mehlstäubler, Nature Commun. 8, 15364 (2017).

[73] P. Schiller and A. Seeger, physica status solidi b 3, K122-K127 (1963).

[74] D.E. Makarov, J. Chem. Phys. 144, 030901 (2016).

[75] W. Quapp and J.M. Bofill, J. Comput. Chem. 37, 2467-2478 (2016).

[76] W. Quapp and J.M. Bofill, Int. J. Quantum Chem. 118, e25522 (2018).

[77] A. Maitra and G. Arya, Phys. Chem. Chem. Phys. 13, 1836-1842 (2011).

[78] W. Quapp, J.M. Bofill and J. Ribas-Ariño, Int. J. Quantum Chem. 118 e25775 (2018).

[79] M. Basilevsky and A. Shamov, Chem. Phys. 60, 347-358 (1981).

[80] D.K. Hoffmann, R.S. Nord and K. Ruedenberg, Theor. Chim. Acta 69, 265-280 (1986).

[81] W. Quapp, Theor. Chim. Acta 75, 447-460 (1989).

[82] H.B. Schlegel, Theor. Chim. Acta 83, 15- 20 (1992).

[83] J.Q. Sun and K. Ruedenberg, J. Chem. Phys. 98, 9707-9714 (1993).

[84] M. Hirsch and W. Quapp, Chem. Phys. Lett. 395 (1-3), 150-156 (2004).

[85] J.M. Bofill, W. Quapp and M. Caballero, J. Chem. Theory Comput. 8, 927-935 (2012). 
[86] J.M. Bofill, J. Ribas-Ariño, S.P. García and W. Quapp, J. Chem. Phys. 147, 152710 (2017).

[87] D. Gangloff, A. Bylinskii, I. Counts, W. Jhe and V. Vuletić, Nat. Phys. 11, 915 - 919 (2015).

[88] K. Fukui, J. Phys. Chem. 74, 4161-4163 (1970).

[89] W. Quapp and D. Heidrich, Theor. Chim. Acta 66, 245-260 (1984).

[90] O.M. Braun, Y.S. Kivshar and I.I. Zelenskaya, Phys. Rev. B 41, 7118 (1990).

[91] S.N. Coppersmith and D.S. Fisher, Phys. Rev. B 28, 2566- 2581 (1983).

[92] J.S. Shie and K.M. Hsiao, Phys. Rev. B 24, 3231-3238 (1981).

[93] A. Seeger, Proc. Roy. Soc. London A 371, 173 (1980).

[94] R. Thom, Structural Stability and Morphogenesis: An Outline of a General Theory of Models (Addison-Wesley, Reading, MA, 1989).

[95] D. Heidrich, W. Kliesch and W. Quapp, Properties of Chemically Interesting Potential Energy Surfaces (Springer, Berlin, Heidelberg, 1991).

[96] L. Karpa, A. Bylinskii, D. Gangloff, M. Cetina and V. Vuletić, Phys. Rev. Lett. 111, $163002(2013)$.

[97] O.M. Braun, Y.S. Kivshar and M. Peyrard, Phys. Rev. E 56 (5), 6050 (1997).

[98] S. Zhang, S. Qi, L.I. Klushin, A.M. Skvortsov, D. Yan and F. Schmid, J. Chem. Phys. 148, 044903 (2018).

[99] Y. Braiman, J. Baumgarten and J. Klafter, Phys. Rev. B 47, 11159-11166 (1993).

[100] W. Quapp and B. Schmidt, Theor. Chem. Acc. 128, 47-61 (2011).

[101] B. Schmidt and W. Quapp, Theor. Chem. Acc. 132, 1305-1313 (2012).

[102] S. Aubry, Phys. D 103, 201-250 (1997).

[103] M. Meister and L.M. Floria, Eur. Phys. J. B 37, 213-221 (2004).

[104] S. Aubry, J. Phys. France 44, 147-162 (1983).

[105] B. Hu and J. Shi, Physica D 71, 23-38 (1994).

[106] W. Quapp, Chem. Phys. Lett. 253 (3-4), 286-292 (1996).

[107] T. Pruttivarasin, M. Ramm, I. Talukdar, A. Kreuter and H. Haeffner, New J. Phys. 13, $075012(2011)$.

[108] M. Weiss and F.J. Elmer, Z. Physik B Cond. Matter 69, 55-69 (1997). 


\section{Newton Trajectories for the tilted Frenkel-Kontorova Model}

W. Quapp, ${ }^{\mathrm{a}}$ and J. M. Bofill ${ }^{\mathrm{b}}$

${ }^{a}$ Mathematisches Institut, Universität Leipzig, PF 100920, D-04009 Leipzig, Germany Email: quapp@uni-leipzig.de

bepartament de Química Inorgànica i Orgànica, Secció de Química Orgànica, and Institut de Química Teòrica i Computacional, (IQTCUB), Universitat de Barcelona, Martí i Franquès 1, 08028 Barcelona, Spain

Email: jmbofill@ub.edu

\section{Supplementary Material}

xxxxxxxxxxxxxx SM xxxxxxxxxxxxxxxxx

1. Data of the discussed structures of the 23-atomic chain

1.1. a) The 'left' minimum is at energy 19.824175 .

The structure is shown in the text.

Coordinates

$\begin{array}{lllll}0.3010 & 4.78629 & 8.2743 & 12.6753 & 17.1849 \\ 20.699 & 25.1744 & 29.6916 & 33.2206 & 37.7223 \\ 42.2472 & 45.7856 & 50.297 & 54.84 & 58.3925 \\ 62.908 & 67.4994 & 71.0919 & 75.6031 & 80.3176 \\ 84.0536 & 88.4852 & 93.4143 & & \end{array}$

The 5 lowest eigenvalues are:

$0.3565,0.2293,0.1675,0.1132,0.0000402$.

The lowest eigenvector is

$0.0309,0.0600,0.0987,0.0851,0.1543$,

$0.2454,0.1913,0.3163,0.5759,0.3015$,

$0.2499,0.4041,0.2325,0.128,0.1472$,

$0.0977,0.0505,0.0533,0.0368,0.0193$,

$0.0207,0.0131,0.0067$

\section{1. b) The 'right' minimum is}

Coordinates

$0.8314,5.7595,10.188,13.9256,18.6413$ $23.1508,26.744,31.3368,35.8509,39.4036$, $43.9479,48.4581,51.9966,56.5227,61.0232$, $64.5523,69.0706,73.5450,77.0593,81.5701$, $85.9700,89.4589,93.9452$

Its structure is shown in the text. 


\subsection{The symmetric $S P_{1}$ between the two global minimums}

Coordinates

$0.324, \quad 4.832,8.346,12.742,17.313$,

$20.884,25.349,30.03,33.728,38.163$,

$43.046,47.124,51.202,56.085,60.520$,

$64.218,68.898,73.364,76.935,81.506$,

$85.902,89.416,93.924$

Its energy is 19.96437. Its structure is shown in the text. Its first eigenvalues are: $-0.0265,0.0109,0.0725,0.1598$. The negative eigenvector is not symmetric

$0.1017,0.1813,0.3995,0.2512,0.2277$,

$0.4063,0.2626,0.2062,0.3409,0.2239$,

$0.1672,0.2676,0.1747,0.1254,0.1945$,

$0.125,0.0861,0.1289,0.0797,0.0512$,

$0.0713,0.0396,0.0204$

\section{3. a) $B B P$ on the NT to direction $(1, . ., 1)^{T}$}

The left BBP point is at energy 19.88 with the gradient norm 0.087 .

$0.372, \quad 4.907,8.446,12.794,17.349$,

$20.894,25.307,29.873,33.428,37.865$,

$42.445,46.016,50.459,55.072,58.679$,

$63.112,67.801,71.510,75.881,80.695$,

$84.660,88.772,93.59$

\section{3. b) The right $B B P$ point}

It is at energy 19.8725 with the gradient norm 0.08 .

$0.657,5.473,9.579,13.552,18.378$,

$22.761,26.461,31.156,35.606,39.198$,

$43.812,48.269,51.826,56.406,60.856$,

$64.397,68.963,73.389,76.921,81.476$,

$85.839,89.359,93.886$

\subsection{A second $S P_{1}$ between the two minimums}

Coordinates

$0.782, \quad 5.675,9.997,13.777,18.494$,

$22.862,26.465,31.040,35.249,38.82$,

$43.291,47.124,50.957,55.428,58.999$,

$63.207,67.783,71.386,75.754,80.470$,

$84.251,88.573,93.466$

Its energy is 20.1993. Its symmetric structure is shown in the text. The lowest eigenvalues are $0.1814,0.0756,0.0488,-0.0278$. 
The negative eigenvector is

$0.025,0.0434,0.0987,0.0737,0.0768$,

$0.1539,0.1361,0.1543,0.3204,0.2485$,

$0.2916,0.5674,0.2916,0.2485,0.3204$,

$0.1543,0.1361,0.1539,0.0768,0.0737$,

$0.0987,0.0434,0.025$

\subsection{The $S P_{2}$ in between}

It is at an energy of 20.2139 units.

$\begin{array}{rrrrr}0.6289 & 5.4070 & 9.4167 & 13.4345 & 18.2154 \\ 22.4039 & 26.1912 & 30.8502 & 34.9731 & 38.6923 \\ 43.2493 & 47.1371 & 51.0117 & 55.5652 & 59.2863 \\ 63.4005 & 68.0531 & 71.8323 & 76.0233 & 80.7994 \\ 84.8035 & 88.8271 & 93.6102 & & \end{array}$

Its structure is shown in the text.

Its lowest eigenvalues are $0.1924,0.1002,0.0067,-0.0366,-0.07249$.

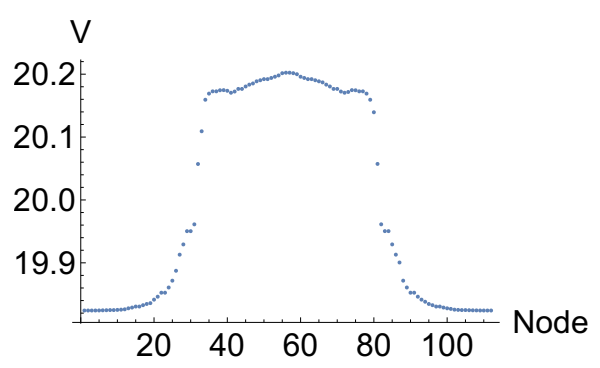

Figure 1. Energy profile over a singular NT connecting the two asymmetric global minimums over a maximum which corresponds to the symmetric $\mathrm{SP}_{2}$.

\section{Programming}

The used parameter of the Fortran program:

$\mathrm{N}=23, \mathrm{Eps}=1.0^{*} \mathrm{E}-06$, ItMax $=7$.

Eps is the convergence criterion of the corrector step.

ItMax is the maximal number of corrector steps.

The norm of the corrector step is maximally 1.0. The corrector sometimes proposes larger steps: they are shorten automatically to 1.0 norm. (Nevertheless the program sometimes goes wrong.)

The number $L$ of nodes was varied, from 65 up to some hundreds, for different aims and versions of the program. (It should be $L=2 \bmod 7$ for an output for the Mma program.) Usually the results are very similar, independent on the number of nodes. Because for special points the Mma optimization and the Fortran program for NTs result in identical values, one can assume that both are correct; as long as the function and the gradient are treated. Our conclusions are arguable. 


\section{Cooperation}

A set of further SPs was detected. They are reported in a further paper. We would like to exchange the data with interested workers. It concerns, as well, the programs (Fortran, and Mathematica). 